\title{
Weaning from Mechanical Ventilation: On the Improvement of the Prediction of Patients' Readiness with Cardiopulmonary Coupling Indices
}

\section{Pablo Armañac-Julián ( $\sim$ parmanac@unizar.es )}

Biomedical Signal Interpretation and Computational Simulation (BSICoS) group at the Aragón Institute of Engineering Research (I3A), IIS Aragón, University of Zaragoza

\section{David Hernando}

Biomedical Signal Interpretation and Computational Simulation (BSICoS) group at the Aragón Institute of Engineering Research (I3A), IIS Aragón, University of Zaragoza

\section{Jesús Lázaro}

Biomedical Signal Interpretation and Computational Simulation (BSICoS) group at the Aragón Institute of Engineering Research (I3A), IIS Aragón, University of Zaragoza

\section{Candelaria de Haro}

Critical Care Center, Hospital Universitari Parc Taulí, Institut d'Investigació Parc Taulí I3PT, Universitat Autónoma de Barcelona, Sabadell

\section{Rudys Magrans}

Better Care, Barcelona

\section{John Morales}

Department of Electrical Engineering-ESAT, STADIUS Center for Dynamical Systems, Signal Processing and Data Analytics, KU Leuven

\section{Jonathan Moeyersons}

Department of Electrical Engineering-ESAT, STADIUS Center for Dynamical Systems, Signal Processing and Data Analytics, KU Leuven

\section{Leonardo Sarlabous}

Critical Care Center, Hospital Universitari Parc Taulí, Institut d'Investigació Parc Taulí I3PT, Universitat Autónoma de Barcelona, Sabadell

\section{Josefina López-Aguilar}

Critical Care Center, Hospital Universitari Parc Taulí, Institut d'Investigació Parc Taulí I3PT, Universitat Autónoma de Barcelona, Sabadell

\section{Carles Subirà}

Department of Intensive Care, Fundació Althaia, Universitat Internacional de Catalunya, Manresa

\section{Rafael Fernández}

Department of Intensive Care, Fundació Althaia, Universitat Internacional de Catalunya, Manresa

\section{Michele Orini}


Institute of Cardiovascular Science, University College London

\section{Pablo Laguna}

Biomedical Signal Interpretation and Computational Simulation (BSICoS) group at the Aragón Institute of Engineering Research (I3A), IIS Aragón, University of Zaragoza

\section{Carolina Varon}

Department of Electrical Engineering-ESAT, STADIUS Center for Dynamical Systems, Signal Processing and Data Analytics, KU Leuven

\section{Eduardo Gil}

Biomedical Signal Interpretation and Computational Simulation (BSICoS) group at the Aragón Institute of Engineering Research (I3A), IIS Aragón, University of Zaragoza

\section{Raquel Bailón}

Biomedical Signal Interpretation and Computational Simulation (BSICoS) group at the Aragón Institute of Engineering Research (I3A), IIS Aragón, University of Zaragoza

\section{Lluís Blanch}

Critical Care Center, Hospital Universitari Parc Taulí, Institut d'Investigació Parc Taulí I3PT, Universitat Autónoma de Barcelona, Sabadell

\section{Research Article}

Keywords: ICU, SBT, CPC, HRV

Posted Date: December 28th, 2020

DOI: https://doi.org/10.21203/rs.3.rs-122028/v1

License: (9) (i) This work is licensed under a Creative Commons Attribution 4.0 International License. Read Full License

Version of Record: A version of this preprint was published at Scientific Reports on August 6th, 2021. See the published version at https://doi.org/10.1038/s41598-021-95282-2. 


\title{
Weaning from Mechanical Ventilation: On the Improvement of the Prediction of Patients' Readiness with Cardiopulmonary Coupling Indices
}

\author{
Pablo Armañac-Julián ${ }^{1,2, *}$, David Hernando ${ }^{1,2}$, Jesús Lázaro ${ }^{1,2}$, Candelaria de Haro ${ }^{3,4}$, \\ Rudys Magrans ${ }^{5}$, John Morales $^{6}$, Jonathan Moeyersons ${ }^{6}$, Leonardo Sarlabous ${ }^{3}$, \\ Josefina López-Aguilar ${ }^{3,4}$, Carles Subiràn, Rafael Fernández ${ }^{4,7}$, Michele Orini ${ }^{8,9}$, Pablo \\ Laguna $^{1,2}$, Carolina Varon ${ }^{6,10}$, Eduardo Gil ${ }^{1,2}$, Raquel Bailón ${ }^{1,2}$, and Lluís Blanch ${ }^{3,4}$
}

\author{
${ }^{1}$ Biomedical Signal Interpretation and Computational Simulation (BSICoS) group at the Aragón Institute of \\ Engineering Research (I3A), IIS Aragón, University of Zaragoza, Spain. \\ ${ }^{2}$ CIBER de Bioingeniería, Biomateriales y Nanomedicina (CIBER-BBN), Instituto de Salud Carlos III, Madrid, Spain. \\ ${ }^{3}$ Critical Care Center, Hospital Universitari Parc Taulí, Institut d'Investigació Parc Taulí I3PT, Universitat Autónoma \\ de Barcelona, Sabadell, Spain. \\ ${ }^{4}$ CIBER de Enfermedades Respiratorias (CIBER-ES), Instituto de Salud Carlos III, Madrid, Spain. \\ ${ }^{5}$ Better Care, Barcelona, Spain. \\ ${ }^{6}$ Department of Electrical Engineering-ESAT, STADIUS Center for Dynamical Systems, Signal Processing and Data \\ Analytics, KU Leuven, Belgium. \\ ${ }^{7}$ Department of Intensive Care, Fundació Althaia, Universitat Internacional de Catalunya, Manresa, Spain \\ ${ }^{8}$ Institute of Cardiovascular Science, University College London, London, U.K. \\ ${ }^{9}$ Barts Heart Centre, St Bartholomews Hospital, University College London,U.K. \\ ${ }^{10}$ Circuits and Systems (CAS) group, Delft University of Technology, the Netherlands. \\ *parmanac@unizar.es
}

\section{ABSTRACT}

The ideal moment to withdraw respiratory supply of patients under Mechanical Ventilation (MV) at Intensive Care Units (ICU), is not easy to be determined for clinicians. Although the Spontaneous Breathing Trial (SBT) provides a measure of the patients' readiness, there is still around $15-20 \%$ of predictive failure rate. This work explores both Heart Rate Variability (HRV) and Cardiopulmonary Coupling (CPC) estimates as complementary information for readiness prediction. The CPC is related to how the mechanisms regulating respiration and cardiac pumping are working simultaneously, and it is defined from HRV in combination with respiratory information. Three different techniques are used to measure CPC, including Orthogonal Subspace Projections, Dynamic Mutual Information and Time-Frequency Coherence.

22 patients undergoing SBT in pressure support ventilation are analysed in the 24 hours previous to the SBT. 13 had a successful weaning and 9 failed the SBT or needed reintubation -being both considered as failed weaning. Results illustrate that traditional variables such as heart rate, respiratory frequency, and the parameters derived from HRV do not differ in patients with successful or failed weaning. However, significant statistical differences are found for the novel CPC parameters, throughout the whole recordings, comparing the values of the two groups. In addition, the night prior to SBT is the moment where differences are higher, probably because patients with failed weaning might be experiencing more respiratory episodes, e.g. apneas during the night, which is directly related to a reduced RSA. Therefore, results suggest that the traditional measures could be used in combination with these novel CPC biomarkers to help clinicians better predict if patients are ready to be weaned.

\section{Introduction}

Most of the patients admitted to Intensive Care Units (ICU) present symptoms of respiratory failure and need the support of Mechanical Ventilation (MV). MV is a procedure of artificial respiration that supplies or collaborates with a patient's respiratory function, in order to have an efficient gas exchange and reduce the respiratory effort. As the respiratory muscles and the nervous system recover, patients are prepared to maintain normal breathing autonomously, making the respiratory support unnecessary.

This process of withdrawing MV is known as "Weaning" and is a challenging and very delicate procedure. The American Thoracic Society (ATS) and the American College of Chest Physicians (CHEST) identify weaning as an investigation priority 
and they address some recommendations for the process of liberation from $\mathrm{MV}^{1}$. The international consensus conference in $2007^{2}$, recommends that weaning should be considered as soon as possible. However, weaning failure and reintubation, due to premature liberation, have been significantly associated with increased mortality, reported in the range from $25 \%$ to $50 \%{ }^{3-9}$. This could lead to dyspnea and hypoxemia $3,10,11$. Reintubation after a premature weaning can lead to an increased risk of severe respiratory complications such as ventilator induced lung injury (VILI) ${ }^{12}$, ventilator-associated pneumonia $(\text { VAP) })^{5,13}$, or ventilator induced diaphragmatic dysfunction ${ }^{14,15}$. Prolonged MV also leads to VILI, barotraumas and VAP ${ }^{2}$

Once the patient is deemed ready for weaning, the Spontaneous Breathing Trial (SBT) assesses the patient's ability to breathe independently ${ }^{2}$. The SBT is, currently, the best diagnostic test to determine weaning readiness and it provides the clinicians some indicators to determine more accurately if the weaning attempt will be successful or not. It is carried out for nearly 30 minutes, by a low-level inspiratory pressure support (with minimal ventilator support) or a T-tube test with no ventilator support ${ }^{13,16-18}$. It is mainly based on respiratory and hemodynamic parameters like respiratory rate $\left(\mathrm{F}_{r}\right)$, Heart Rate (HR), arterial oxygen saturation $\left(\mathrm{SpO}_{2}\right)$, systolic blood pressure (SBP) and the clinicians' expertise and observation ${ }^{19}$. However, although these parameters are crucial for treating critically ill patients, they are not always reliable predictors for the final weaning decision maybe since they do not consider the physiological interactions of the different regulatory mechanisms ${ }^{13}$. Literature shows that about $15-20 \%$ of patients who pass SBT do not respond to the weaning and they need to be reintubated within the next 48 hours, causing patient suffering, and increasing complications and comorbidities ${ }^{20,21}$.

The cardiovascular control involves several mechanisms to dynamically couple arterial pressure, HR and respiration. This cross-talk between heart, lungs and brain is a complex regulating system mainly coordinated by the Autonomic Nervous System $(\mathrm{ANS})^{22,23}$. Recent clinical and electrophysiological studies revealed a high incidence of autonomic dysfunction in patients admitted to the ICU ${ }^{24,25}$, which has a negative impact on clinical prognosis and increases mortality rates ${ }^{26}$.

Heart rate variability (HRV) is a non-invasive measurement of the ANS function, and it has been used as a tool to compare the outcome of patients who went through a weaning procedure. Patients who failed SBT were observed to have reduced HRV during SBT compared to after weaning ${ }^{27,28}$. Previous studies tried to analyse the state of the patients in the 30 minutes after the SBT using HRV indicators. The study in ${ }^{29}$ demonstrates the added value of HRV and respiration rate variability, comparing the outcomes in the 30 minutes during the SBT. They found that altered HRV and respiration rate variability during the SBT are associated with a weaning failure. Other studies like $\mathrm{er}^{31}$ also study the HRV and derived indexes right before, during and after the SBT to classify patients and contribute to the clinical decision of weaning. However, all the previous studies were observational. None of them analysed the role of the ANS in long-term and, moreover, no previous studies looked for differences only before the SBT, in order to improve the predictive value of the indices for weaning readiness.

In addition to HRV, another important mechanism known as Cardiopulmonary Coupling (CPC) can be derived if HRV is combined with respiratory information ${ }^{32,33}$. The CPC is mainly expressed through the Respiratory Sinus Arrhythmia (RSA) ${ }^{34}$, which is the acceleration and deceleration of the heart driven by respiration. The physiological role is still a matter of debate, but strong CPC is postulated to enhance the pulmonary gas exchange, improve cardiac efficiency, maintain physiological levels of arterial $\mathrm{CO}_{2}$ and stabilize arterial blood pressure ${ }^{35}$. Although a lot of discussion exists on which mechanism is responsible for $\mathrm{RSA}^{36-38}$, studies suggest that the ANS, via the vagus nerve, plays the major role in it, together with some mechanical effects and the baroreflex. Then, since state-of-the-art studies use the CPC as an ambulatory biomarker of sleep quality ${ }^{39,40}$, as a predictor for early treatment response in depressed patients ${ }^{41}$, or for asthma evolution monitoring in children ${ }^{42}$, in this work it is hypothesized that these estimators of the CPC could also respond to the multi-factorial nature of the weaning process.

This study aims to find new biomarkers that could help clinicians better predict if a patient is ready to be weaned. To this end, the reliability of HRV and, specially, CPC estimators will be assessed. The influence of the timing for the analysis is evaluated both along the day and related to the moment when the SBT is performed.

Different approaches exist to quantify the strength of the RSA for CPC assessment, among which the following stand out: analysis of the HRV in the High Frequency (HF) band ${ }^{43}$, Time Frequency Coherence Analysis (TFC) ${ }^{44}$, Information Dynamics (ID) ${ }^{45}$, Orthogonal Subspace Projections (OSP) ${ }^{46}$, Bivariate Phase Rectified Signal Averaging ${ }^{47}$, Pole Specific Spectral Causality ${ }^{48}$, and Cardiopulmonary Phase Synchronization ${ }^{49}$. The performance of these estimators of CPC is studied in $^{50}$, and the indices derived from TFC, ID and OSP are the ones used in this work.

\section{Materials}

\section{Database}

The database was constructed prospectively at two hospitals in Spain, in the Hospital Universitari Parc Taulí and the Fundació Althaia, using the connectivity platform Better Care (Better Care, Barcelona, Spain. US patent No. 12/538,940). This database was aimed to establish a new model for the prediction of successful weaning. The Institutional Review Boards of Comitè d'Ética d'Investigació amb medicaments at the Corporació Sanitària Parc Taulí and the Clinical Research Ethics Committee of Fundació Unió Catalana d'Hospitals approved the database and the study protocol. The requirement for informed consent was waived as part of the study approval, since the current study was an ancillary analysis. Therefore, all the signals were 


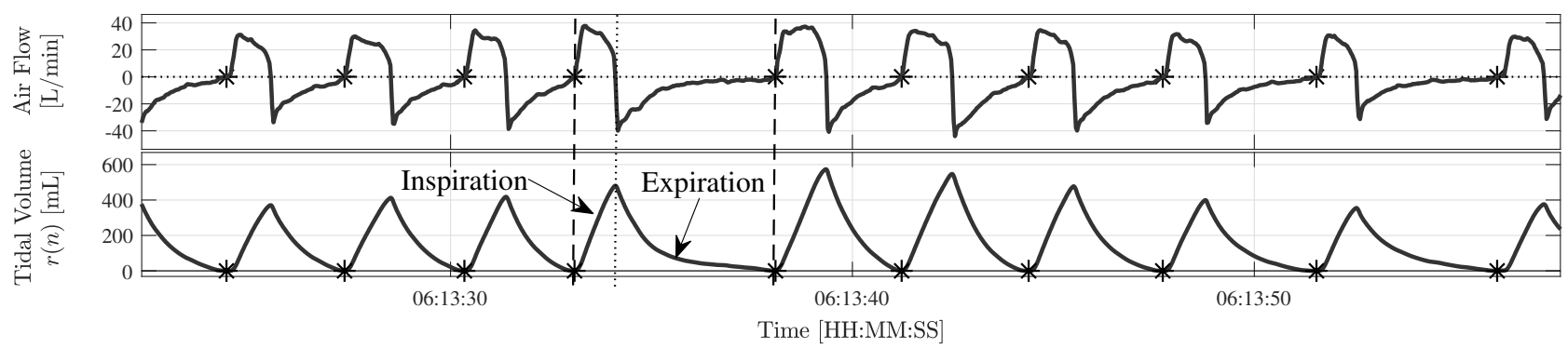

Figure 1. Example of respiration in PSV mode. The airflow signal is plotted at the (top) and the derived tidal volume signal at the (bottom). The onset of inspiration, $n_{\mathrm{I}, \mathrm{ON}}$, is marked with asterisks.

anonymous and encrypted to ensure privacy. The guidelines followed in this study were according to the applicable Spanish regulations (Biomedical Research Law 14/2007).

Patients with neurological disorder, dementia or focal brain injury at ICU admission were excluded from the analysis. In addition, only those patients ventilated with assist/support ventilation modes were considered, i.e., excluding patients that were in controlled ventilation modes in the 24 hours previous to the SBT. Data during the 24 hours prior to the SBT were obtained from 22 patients. For this subset, the most common MV mode was Pressure Support Ventilation (PSV), but also some patients spent some time in Continuous Positive Airway Pressure (CPAP). Excluding controlled-MV modes implies that respiration may be more irregular, and apnoea or periodic breathing could happen. An example of the respiratory pattern in PSV mode is shown in Figure 1.

\section{Criteria for weaning readiness}

The following criteria were used to determine if a patient was presumably ready to be weaned, i.e., if a patient was ready to perform the $\mathrm{SBT}^{19,20}$ :

1. Medical Assessment

- Improvement or recovery of the cause for MV

- Adequate cough

- Absence of secretion

- No neuromuscular blocking agents

2. Parametric Measures

- Time in MV > 24 hours

- $H R<140 \mathrm{bpm}$

- $S B P \in[90,160] \mathrm{mmHg}$

- Haemoglobin $(\mathrm{Hb}) \geq 8 \mathrm{~g} / \mathrm{dL}$

- $\mathrm{SpO}_{2}>90 \%$, with $\mathrm{FiO}_{2} \leq 40 \%$

- Tidal Volume $\left(\mathrm{V}_{T}\right)>5 \mathrm{~mL} \cdot \mathrm{kg}$

- $F_{r}<35 \mathrm{rpm}$

- $\mathrm{pH} \geq 7.30$ (no respiratory acidosis)

- Body Temperature $(\mathrm{T}) \in[35,38]^{\circ} \mathrm{C}$

- $\mathrm{PEEP}<8 \mathrm{cmH}_{2} \mathrm{O}$

- Maximal Inspiratory Pressure (MIP) $\leq-25 \mathrm{cmH}_{2} \mathrm{O}$

- Rapid Shallow Breathing Index (RSBI) $<105$

- Richmond Agitation-Sedation Scale (RASS): -1/0

- Glasgow Coma Scale (GCS) >8

- Minimal vasopressors or inotropes $<5 \mu \mathrm{gr} / \mathrm{kg} / \mathrm{minute}$ (stable cardiovascular status)

\section{Criteria for successful SBT}

Subsequently, patients presumably ready to be weaned, had to perform the SBT. The SBT was carried out by a low-level inspiratory pressure support or by a T-tube test. The criteria listed in this section show the indicators used to evaluate the success of the SBT, in order to decide if MV could finally be withdrawn.

The following criteria were used to assess if SBT was successful ${ }^{19}$ :

- Good tolerance to the SBT

- $F_{r}<35 \mathrm{rpm}$

- $\mathrm{HR}<140$ bpm 
- $\mathrm{HR}<20 \%$ change from baseline

- $\mathrm{SpO}_{2}>90 \%$ or $\mathrm{PaO}_{2}>60 \mathrm{mmHg}$ on $\mathrm{FiO}_{2}<40 \%$

- $\mathrm{SBP} \in[80,180] \mathrm{mmHg}$

- $\mathrm{SBP}<20 \%$ change from baseline

- No signs of increased work of breathing or distress

\section{Patient classification and demographics}

When the health condition of a patient improved enough, they are probably ready for weaning ${ }^{19,20}$. Then, these patients must perform the SBT. Patients presenting at least one item of the intolerance criteria for successful $\mathrm{SBT}^{19}$, were not ready for discontinuation and weaning failure was considered. These patients belong to the $F$-group. Patients who passed SBT ${ }^{19}$, belong to the $S$-group. However, 2 patients that passed the SBT required orotracheal intubation or reconnection to non-invasive MV within 48 hours after SBT. These 2 patients were reclassified in the F-group. Refer to Fig. 2 for the patient classification scheme.

With all these premises, there are 13 patients in the $S$-group and 9 in the F-group. Accordingly, for the analysed subset, actual weaning readiness is for the $54 \%$ of patients, and the SBT was incorrect for the $13 \%$ of the tests that passed.

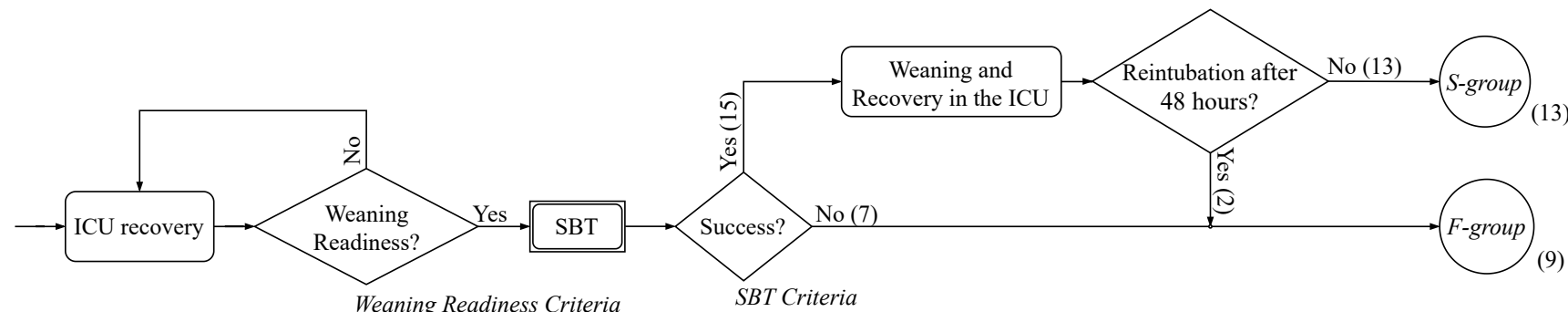

Figure 2. Algorithm for the definition of the weaning success. Patients are classified into the $S$-group or $F$-group after the Spontaneous Breathing Trial (SBT). The $S$-group stands for the group of patients successfully weaned (successful SBT and no need of reintubation). F-group stands for the group of patients with SBT failure and patients with SBT success but with the need of reintubation after 48 hours of weaning. Numbers in parentheses represent the number of patients according to the respective criteria.

The demographics of patients are summarized in Table 1. The variables available include: age, gender, Acute Physiology and Chronic Health Evaluation II (APACHE II), Sequential Organ Failure Assessment (SOFA), reason for MV, MV duration, ICU length of stay, ICU mortality and In-hospital mortality.

Table 1. Demographics. Data are presented as median [IQR 25-75] and percentages.

\begin{tabular}{lll}
\hline & \multicolumn{1}{c}{$\boldsymbol{S}$-group } & F-group \\
\cline { 2 - 3 } & $(\mathrm{N}=13)$ & $(\mathrm{N}=9)$ \\
\hline Age (years) & $65[60-72]$ & $69[59-72]$ \\
Gender (\% female) & $15 \%$ & $33 \%$ \\
APACHE II at admission & $18[14-23]$ & $16[10-20]$ \\
SOFA at admission & $7[6-8]$ & $6[3-10]$ \\
\hline Reason for MV : & & \\
$\quad$ - Acute Respiratory Failure & $31 \%$ & $22 \%$ \\
$\quad$ Sepsis & $38 \%$ & $33 \%$ \\
• Neurologic & $8 \%$ & $11 \%$ \\
• Other & $23 \%$ & $34 \%$ \\
\hline MV duration (days) & $6[4-10]$ & $12[8-16]$ \\
ICU length of stay (days) & $8[6-12]$ & $18[13-23]$ \\
ICU mortality & $8 \%$ & $22 \%$ \\
In-hospital mortality & $8 \%$ & $22 \%$ \\
\hline
\end{tabular}

APACHE: Acute Physiology and Chronic Health Evaluation; SOFA: Sequential Organ Failure Assessment; MV: Mechanical Ventilation; ICU: Intensive Care Unit. 


\section{Data acquisition and data analysis}

Physiological signals were continuously recorded using the connectivity platform Better Care ${ }^{51}$. The Better Care system (Better Care, Barcelona, Spain. US patent No. 12/538,940), is a proprietary system for data collection designed to interact with output signals from mechanical ventilators and bedside monitors rather than directly with patients. It was firstly developed to interoperate signals from different ventilators and monitors, and subsequently compute algorithms for diagnosing patientventilator asynchronies (ClinicalTrial.gov, NCT03451461).

Better Care standardizes, synchronizes and stores the signals of all the bedside monitors and ventilators at 200 samples per second, from intubation in the ICU to liberation from MV. It uses drivers specifically designed to interact with output signals from mechanical ventilators and bedside monitors rather than directly with patients. Different biomedical signals were recorded, including the three bipolar leads of the electrocardiogram (ECG), as well as the respiratory signals: air flow and airway pressure. In addition, pulse photoplethysmography, blood pressure via invasive catheter, and $\mathrm{SpO}_{2}$ were also recorded.

The onset of inspiration for each breath, $i$, labelled as $n_{\mathrm{I}, \mathrm{ON}_{i}}$, was delineated using an algorithm implemented in the Better Care platform. For each respiratory cycle, information on the type of ventilation mode, the trigger of respiration and the appearance or absence of asynchronies, such as ineffective efforts or double cycling, were also given ${ }^{51,52}$. Notice that PSV mode forces spontaneous breathing, so no breath was automatically triggered by the MV.

\section{Methods}

Methodologies are based on the signal processing of the ECG and respiration. First, HRV and respiratory information are estimated. Afterwards, the indices of CPC, based on TFC, ID and OSP, can be obtained.

All these different measures are used in this work to characterize the evolution of the ANS function or the CPC mechanisms. However, the algorithms do not extract the information in the same way, and the different indices may have different temporal resolution. For this reason, the average value in consecutive 30-minutes periods is considered for each index, in order to be able to compare all the different estimators through the 24-hour recordings.

\section{HRV and respiratory information estimation}

The baseline-corrected tidal volume signal, $r(t)$, is obtained integrating the instantaneous air flow signal followed by baseline subtraction. The baseline is estimated by modified Akima piecewise cubic Hermite interpolation of the $n_{\mathrm{I}, \mathrm{ON}}$ series estimated at the integrated air flow signal. This ensures that each tidal volume breath begins and ends with zero litres, as depicted in Figure 1. The respiratory frequency signal, $F_{r}(t)$, is derived from the estimation in each breath by computing the inverse of the instantaneous respiration-to-respiration difference, $\mathrm{F}_{r}$, from the $n_{\mathrm{I} \text {,ON }}$ series. After that, $r(t)$ and $F_{r}(t)$ are resampled at $4 \mathrm{~Hz}$, to obtain the tidal volume signal, $r(n)$, and the respiratory frequency signal, $F_{r}(n)$, respectively.

The lead II of the ECG is upsampled at $1000 \mathrm{~Hz}$ with cubic spline interpolation, to ensure that HRV analysis is not compromised by the effect of low sampling frequency ${ }^{53,54}$. Then, the QRS-complexes are detected by means of a waveletbased method ${ }^{55}$. The time between two successive R waves defines the RR interval. Ectopic beats and miss-detections are corrected as described in ${ }^{56}$. The exclusion of non-normal RR intervals, that do not represent the ANS function, results in the normal-to-normal (NN) interval series.

The temporal indices of HRV are calculated from the NN series ${ }^{43}$, and the ones used in this work are the standard deviation of the NN intervals (SDNN), and the root mean-square of successive differences of adjacent intervals (RMSSD). SDNN is the "gold standard" parameter of HRV for medical stratification of cardiac risk, when recorded over a 24-hours period ${ }^{43}$.

Afterwards, the HR is represented to produce a signal which accurately reflects the HRV, so that classical HRV indices in the frequency domain can be derived. In this work, the HRV is estimated using the Time-Varying Integral Pulse Frequency Modulation model (TVIPFM) ${ }^{57}$. Given a particular beat time occurrence series, the instantaneous HR, $d_{\mathrm{HR}}(t)$, can be expressed, from the TVIPFM, as $d_{\mathrm{HR}}(t)=(1+m(t)) / T(t)$. The term $1 / T(t)$ represents the time-varying mean HR and $m(t)$ represents the modulating signal of interest, which is assumed to contain the ANS modulation of the sinoatrial node. The instantaneous mean HR, $d_{\mathrm{HRM}}(t)=1 / T(t)$, is obtained by low-pass filtering $d_{\mathrm{HR}}(t)$ at $0.03 \mathrm{~Hz}$. The $\mathrm{HRV}$ signal is represented by the term $d_{\mathrm{HRV}}(t)=d_{\mathrm{HR}}(t)-d_{\mathrm{HRM}}(t)$. Finally, the modulating signal is estimated as $m(t)=d_{\mathrm{HRV}}(t) / d_{\mathrm{HRM}}(t)$ and the evenly sampled version of the modulating signal, $m(n)$, is obtained by resampling $m(t)$ at $4 \mathrm{~Hz}$.

It is known that respiration affects HRV. Therefore, the frequency domain analysis of the HRV is performed guided by respiration ${ }^{58}$. In order to do so, the High Frequency (HF) band is redefined to be centred at the respiratory frequency: $\Omega_{\mathrm{HF}}^{r}(t)=\left[F_{r}(t)-0.15, F_{r}(t)+0.15\right] \mathrm{Hz}$, and the HF power, $\mathrm{P}_{\mathrm{HF}}$, is defined as the power within this band. Low Frequency (LF) power, $\mathrm{P}_{\mathrm{LF}}$, is defined as the power in the classic LF band ${ }^{43}: \Omega_{\mathrm{LF}}=[0.04,0.15] \mathrm{Hz}$. The use of the modified HF band was also encouraged by the increased $\mathrm{F}_{r}$ observed in some mechanically ventilated patients, who had a respiratory frequency above $24 \mathrm{rpm}$, i.e., $0.4 \mathrm{~Hz}$, that could lead to an underestimation of the power using the classical HF band. The balance between the Sympathetic Nervous System (SNS) and Parasympathetic Nervous System (PNS) activity, the so-called sympathovagal balance $^{59}$, is represented by the power normalized in the $\mathrm{LF}$ band $\mathrm{P}_{\mathrm{LF}}^{n}=\mathrm{P}_{\mathrm{LF}} /\left(\mathrm{P}_{\mathrm{LF}}+\mathrm{P}_{\mathrm{HF}}\right)$. 
Since most of the power of HRV in a 24-hour recording resides in the frequencies below HF and LF power, the Very Low Frequency (VLF) power, $\mathrm{P}_{\mathrm{VLF}}$, is also studied. The $\mathrm{P}_{\mathrm{VLF}}$ is accounted for by fluctuations in NN intervals that have a period larger than 25 seconds: $\Omega_{\mathrm{VLF}}=(0,0.04] \mathrm{Hz}$. The physiological role of $\mathrm{P}_{\mathrm{VLF}}$ is still not clear, but it is believed to be related to the circadian rhythms, core body temperature, and metabolism ${ }^{60}$.

These frequency domain parameters are calculated using a TF distribution belonging to the Cohen's class ${ }^{44}$. A time and frequency resolution of $11.25 \mathrm{~s}$ and $0.039 \mathrm{~Hz}$, respectively, is used.

\section{Time-frequency analysis}

The respiratory influences on HRV can be captured based on TF coherence, which is given by:

$$
\hat{\gamma}(t, f)=\frac{\left|\hat{S}_{r, m}(t, f)\right|}{\sqrt{\hat{S}_{r}(t, f) \hat{S}_{m}(t, f)}},
$$

where $\hat{\gamma}(t, f) \in[0,1] . \hat{S}_{r}(t, f)$ and $\hat{S}_{m}(t, f)$ are the auto-power spectral densities calculated by means of the Cohen's Class Wigner Ville Distribution ${ }^{44}$ of respiration, here represented by its surrogate tidal volume $r(n)$, and HRV, represented by $m(n)$, respectively. $\hat{S}_{r, m}(t, f)$ is the cross-power spectral density. An illustrative example of the TFC performance can be seen in Figure 3.

A significant coherence level of coupling between HRV and respiration is set by the signal-independent threshold, $\gamma_{\mathrm{TH}}(t, f ; \alpha)$. It is established based on a surrogate data analysis ${ }^{44}$, with $\alpha=5 \%$ risk that both signals are coupled when real coupling does not exists $\gamma_{\mathrm{TH}}(t, f ; 0.05)=\gamma_{0}$. The region $\Omega_{\mathrm{HF}}^{r, c}(t, f)$, from which the coherence is estimated, is identified as the region where the TF coherence is significant within the HF band centred at the respiratory frequency, $\Omega_{\mathrm{HF}}^{r}(t)$ :

$$
\Omega_{\mathrm{HF}}^{r, c}(t, f)=\left\{(t, f) \in\left(\mathbb{R}^{+} \times \Omega_{\mathrm{HF}}^{r}(t)\right) \mid \hat{\gamma}(t, f)>\gamma_{0}\right\} .
$$

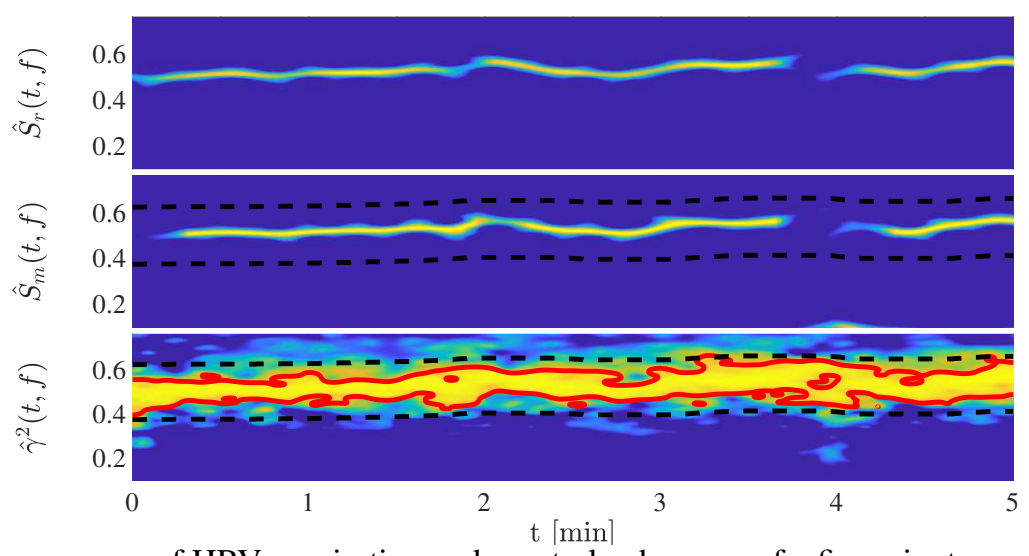

Figure 3. Time-frequency maps of HRV, respiration and spectral coherence of a five minutes period. The TF map of the respiratory signal, $\hat{S}_{r}(t, f)$, is on (top) and the TF map of the HRV modulating signal, $\hat{S}_{m}(t, f)$, is in the (middle). The TF map of the quadratic spectral coherence between respiration and $\operatorname{HRV}, \hat{\gamma}^{2}(t, f)$, is in the (bottom). The white-dotted lines in the TF map of $\hat{S}_{m}(t, f)$ and $\hat{\gamma}^{2}(t, f)$, represent the HF band centred at the respiratory frequency, $\Omega_{\mathrm{HF}}^{r}(t)$. The regions of coherence with statistical significance within the $\mathrm{HF}$ band centred at the $\mathrm{F}_{r}, \Omega_{\mathrm{HF}}^{r, c}(t, f)$, are the red-colored area in the $\hat{\gamma}^{2}(t, f)$ map. For further information, see ${ }^{44}$.

To characterize the temporal evolution of the local coupling between the spectral components of the signals, the index $\mathcal{C}_{\mathrm{HF}}(t)$ is defined as:

$$
\mathcal{C}_{\mathrm{HF}}(t)=\int_{\Omega_{\mathrm{HF}}^{r, c}} \hat{\gamma}(t, f) d f / \int_{\Omega_{\mathrm{HF}}^{r c c}} 1 d f
$$

This index takes into account the magnitude of the local coupling, averaged in the HF band.

Now, if the mean significant coherence, $\mathcal{C}_{\mathrm{HF}}(t)$, is averaged in a period of time, it yields to the definition of $\mathscr{C}_{\mathrm{HF}}$ :

$$
\mathscr{C}_{\mathrm{HF}}=\int \mathcal{C}_{\mathrm{HF}}(t) d t / \int 1 d t
$$


Finally, for all the time course, the existence of significant coupling at any frequency in the whole band $\Omega_{\mathrm{HF}}^{r}(t)$ is identified as:

$$
\mathcal{T}_{\mathrm{HF}}(t)= \begin{cases}1, & \text { if } \Omega_{\mathrm{HF}}^{r, c}(t, f) \neq \emptyset \\ 0, & \text { if } \Omega_{\mathrm{HF}}^{r, c}(t, f)=\emptyset\end{cases}
$$

Once the "mask" $\mathcal{T}_{\mathrm{HF}}(t)$ is defined, the percentage where TFC is significant in a period of time, $\mathscr{T}_{\mathrm{HF}}$, can be defined as:

$$
\mathscr{T}_{\mathrm{HF}}=\int \mathcal{T}_{\mathrm{HF}}(t) d t / \int 1 d t .
$$

The index of CPC used from this framework is calculated using Eq. (3) and Eq. (5). This index is noted as $\mathscr{C}_{\mathrm{HF}}^{\mathcal{T}}$ and is composed taking into account the mean significant coherence averaged in a period of time, $\mathscr{C}_{\mathrm{HF}}$, with the percentage of time where TFC is significant in that same period, $\mathscr{T}_{\mathrm{HF}}$ :

$$
\mathscr{C}_{\mathrm{HF}}^{\mathcal{T}}=\mathscr{C}_{\mathrm{HF}} \cdot \mathscr{T}_{\mathrm{HF}}
$$

As for the estimation of the HRV frequency domain parameters, the same TF maps are used to obtain $\hat{S}_{r}(t, f), \hat{S}_{m}(t, f)$ and $\hat{S}_{r, m}(t, f)$, using the TF distribution belonging to Cohen's class ${ }^{44}$. As mentioned before, the time and frequency resolution is fixed to $11.25 \mathrm{~s}$ and $0.039 \mathrm{~Hz}$, respectively, for the TF distribution.

\section{Dynamic mutual information}

It is known that the RSA defines a causal relationship from respiration to HRV, since respiration drives acceleration/deceleration in the HR. This relationship implies that the uncertainty about HRV, can be resolved not only by knowing itself, but also by taking into account the information transferred from respiration. This resolution of entropy, or uncertainty, can be quantified using measures of predictive information ${ }^{45}$.

Let's denote $r_{n}$ and $m_{n}$ as the scalar random values obtained by sampling the process $r(n)$ and $m(n)$, respectively, at the present time, $n$. The vectors $\mathbf{r}^{-}=[r(n-1), \ldots, r(n-M)]^{T}$, and $\mathbf{m}^{-}=[m(n-1), \ldots, m(n-M)]^{T}$, are defined to describe the whole past of each process, with $M$ the model order.

If the information carried by the HRV is split into components related to respiration and others, the predictive information leads to the definition of the Cross Entropy (CE) term, $\mathscr{C} \mathscr{E}_{\mathrm{r} \leftrightarrow \mathrm{m}}$. This term quantifies the amount of information shared at a certain time, $n$, between the present value of $\mathrm{HRV}, m_{n}$, and the past of respiration, $\mathbf{r}^{-}$:

$$
\mathscr{C} \mathscr{E}_{\mathrm{r} \leftrightarrow \mathrm{m}}=I\left(m_{n} ; \mathbf{r}^{-}\right)=H\left(m_{n}\right)-H\left(m_{n} \mid \mathbf{r}^{-}\right)
$$

where $I(\cdot ; \cdot)$ quantifies the mutual information, $H\left(m_{n}\right)$ expresses the amount of information carried by the process in terms of the average uncertainty about $m_{n}$, the so-called Shannon entropy. $H\left(m_{n} \mid \mathbf{r}^{-}\right)$denotes the conditional entropy and it quantifies the average uncertainty that remains about $m_{n}$ when $\mathbf{r}^{-}$is known ${ }^{45}$.

The computation of $\mathscr{C} \mathscr{E}_{\mathrm{r} \leftrightarrow \mathrm{m}}$ is done using the approach presented $\mathrm{in}^{45}$, using the link between information theory and predictability. It is possible to describe the dynamics of the system using a linear vector autoregressive model.

The model order, $M$, is defined as the minimum amount of delays obtained using both the Minimum Description Length principle and the Akaike Information Criterion. The maximum possible delay is set to 10 seconds in order to avoid over-fitting. The minimum possible delay is set to the period equivalent to the lowest frequency of the respiration bandwidth in order to avoid a too-simple model.

\section{HRV decomposition}

By using subspace projections, the HRV can be decomposed into two different components ${ }^{46}$. First, a component describing all variations of HRV linearly related to respiration is derived. After that, the remainder, namely residual component, describes all dynamics modulated by other mechanisms different from respiration, such as the SNS modulations or other vagal modulators unrelated to respiration, plus the possible non-linear influences of respiration.

Given are the respiratory signal, $r(n)$, and the HRV estimated from the modulating signal, $m(n)$. First of all, the respiratory signal, $r(n)$, and the HRV, $m(n)$, are centred to have zero mean and scaled to have standard deviation equal to one. Now, the vectors $\mathbf{r}=[r(0), r(1), \ldots, r(N-M+1)]^{T}$ and $\mathbf{m}=[m(0), m(1), \ldots, m(N-M+1)]^{T}$ are defined, with $N$ the number of samples in a computational period, and $M$ the number of delays to construct a respiratory subspace. The model order, $M$, is the same used for the $\mathscr{C} \mathscr{E}_{\mathrm{r} \leftrightarrow \mathrm{m}}$ computation (see previous section).

The OSP projects $\mathbf{m}$ onto the subspace $\mathbb{V}$. The matrix $\mathbf{V}$ is constructed as a time-delay embedding of $\mathbf{r}$, that spans the subspace $\mathbb{V}$, using $M$ delays. Therefore, the subspace $\mathbb{V}$ is defined by all variations in $\mathbf{r}$, in order to extract all dynamics of the 
HRV that are linearly related to respiration. Once the matrix $\mathbf{V}$ is constructed, the HRV can be projected onto the respiratory subspace $\mathbb{V}$, by means of the projection matrix $\mathbf{P}$ :

$$
\mathbf{m}_{\mathrm{r}}=\mathbf{P} \mathbf{m},
$$

with the projection matrix, $\mathbf{P}$, obtained from $\mathbf{V}$ as:

$$
\mathbf{P}=\mathbf{V}\left(\mathbf{V}^{T} \mathbf{V}\right)^{-1} \mathbf{V}^{T}
$$

As a result, all dynamics of HRV linearly related to respiration are described in $\mathbf{m}_{\mathbf{r}}$. The orthogonal component, $\mathbf{m}_{\perp}$, computed as the residual, $\mathbf{m}_{\perp}=\mathbf{m}-\mathbf{m}_{\mathrm{r}}$, is explained by all other HR modulators not linearly related to respiration. An example of the HRV decomposition can be seen in Fig.4. It is denoted as $m_{\mathrm{r}}(n)$ and $m_{\perp}(n)$, when it is necessary to refer generically to those vectors in time. $\mathrm{CPC}^{46}$ :

After decomposing the HRV, the relative power of the respiratory component, $\mathscr{P}_{\mathrm{m}_{\mathrm{r}}}$, is computed as an estimate of the

$$
\mathscr{P}_{\mathrm{m}}=\frac{\mathbf{m}_{\mathrm{r}}^{T} \mathbf{m}_{\mathrm{r}}}{\mathbf{m}^{T} \mathbf{m}} .
$$
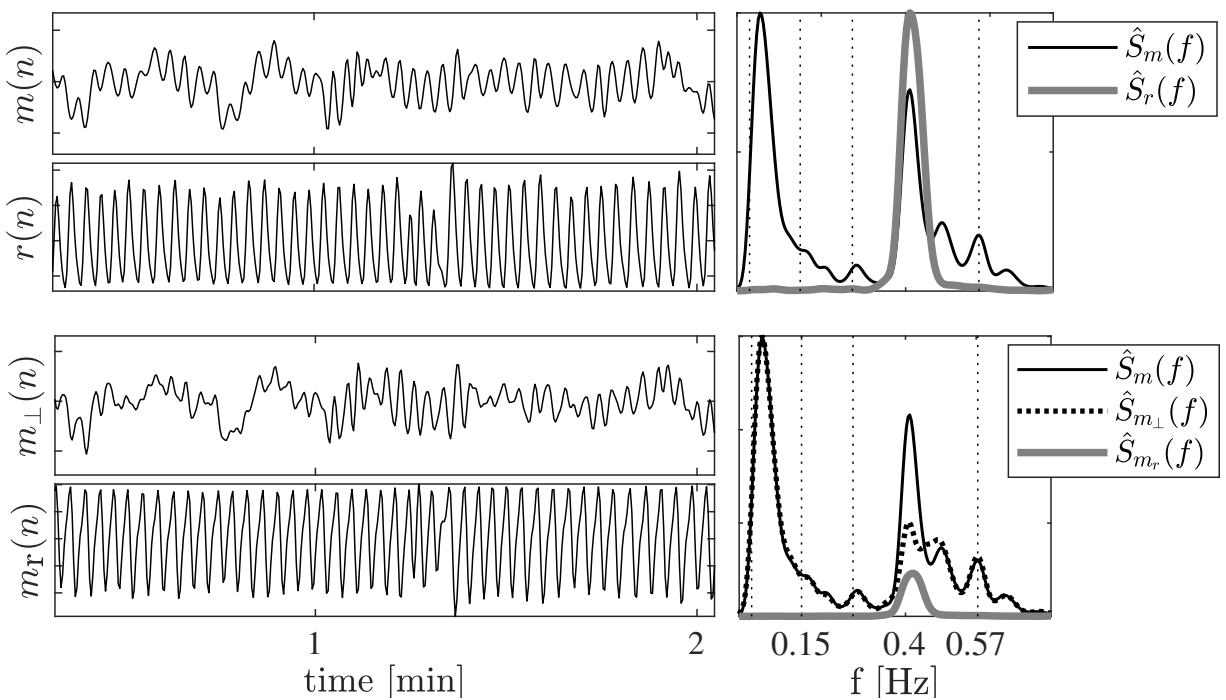

Figure 4. Illustrative example of the OSP decomposition using the HRV and Respiratory signals. The three upper plots are the time evolution of the modulating signal, $m(n)$, the respiratory signal, $r(n)$, and their respective spectra on the upper-right side, $\hat{S}_{m}(f)$ for $m(n)$ and $\hat{S}_{r}(f)$ for $r(n)$. The three plots below represent the OSP decomposition. The respiratory component of $\mathrm{HRV}, m_{r}(n)$, is obtained projecting $m(n)$ onto the respiratory subspace. The modulators of HRV unrelated to respiration are represented in the term $m_{\perp}(n)$. Their corresponding spectra are on the lower-right side. $\hat{S}_{m_{\perp}}(f)$ corresponds to the spectra of $m_{\perp}(n)$, and $\hat{S}_{m_{r}}(f)$ to the spectra of $m_{r}(n)$. For further information, see ${ }^{46}$.

\section{Long-term assessment and statistical analysis}

First, since SDNN is the state-of-art index for medical stratification of cardiac risk in long-term analysis, the SDNN is calculated for the whole period of 24 hours before the SBT. The Mann-Whitney U-test is also used to compare the value for the S-group vs. F-group.

After that, the evolution of the common clinical parameters, HRV and CPC indices, through the 24 hours before SBT are analysed, in order to determine if circadian rhythms could be affecting the regulatory mechanisms and the interpretation of the results. However, it must be considered that the SBT's are generally performed in the morning, but not at the same exact time for all patients. For this reason, all the recordings are segmented from 08:00 p.m. to 10:30 a.m. of the SBT day, so that the same time interval is considered for all the patients. This means that it is being considered only some part of the circadian rhythm.

At this point, the average value in consecutive 30-minutes periods is considered, for the representation of the evolution through the day and for the statistical analysis. To this end, each HRV and CPC indices are calculated separately using different temporal resolutions: 
- The parameters $\mathrm{F}_{r}$ and HR are unevenly sampled at the inspiration onset and heart beats occurrence, respectively. Therefore, the mean value in each half hour is computed.

- For the computation of $\mathscr{C} \mathscr{E}_{\mathrm{r} \leftrightarrow \mathrm{m}}, \mathscr{P}_{\mathrm{m}}$, and the temporal HRV parameters -SDNN and RMSSD-, sliding windows of 3-min-length with $75 \%$ of overlap are used. For these, there is a sample each 45 seconds and a total of 1920 samples in 24 hours. So, the mean of 40 overlapped windows in each 30-minutes period is computed, for each parameter and for each patient.

- Finally, the $\mathscr{C}_{\mathrm{HF}}^{\mathcal{T}}$ and the frequency domain parameters of $\mathrm{HRV}-\mathrm{P}_{\mathrm{VLF}}, \mathrm{P}_{\mathrm{HF}}$ and $\mathrm{P}_{\mathrm{LF}}^{n}-$ are calculated using the TF distribution belonging to Cohen's class ${ }^{44}$. Therefore, these indices are calculated at the resampling frequency, $F_{s}=4 \mathrm{~Hz}$, and thus the average value of 7200 samples, i.e., 30 minutes, is obtained.

Finally, the averaged values for the $S$-group vs. F-group are compared with the non-parametric unpaired Mann-Whitney U-test, for all the parameters. Differences are considered significant for a level of $p \leq 0.05$.

\section{Results}

Table 2 shows the median and quartiles 1 and 3 of the SDNN, calculated in the whole recordings of 24 hours. Higher SDNN is visible for the $S$-group patients, and although the difference is not significant, the $p$-value approaches 0.05 . As expected, the SDNN values are higher considering the 24-hours recordings (see Tab.2), than considering the averaged 3-minutes windows (see Fig.6), for the same group of patients. The evolution of the patients throughout the day before SBT, from 08:00 p.m. to 10:30 a.m., are illustrated in the Figs. 5, 6 and 7. The commonly-used clinical variables respiratory frequency, $\mathrm{F}_{r}$, and heart rate, HR, -shown in Fig.5-, can be compared to the parameters of HRV -in Fig.6- and the CPC estimators -in Fig.7.

Looking at Fig.5, both $\mathrm{F}_{r}$ and $\mathrm{HR}$ rely within the limits of criteria for weaning readiness. In general, patients of the $F$-group have little higher HR and $\mathrm{F}_{r}$. The $\mathrm{F}_{r}$ is significantly higher only at 9:00, moment when HR differences are larger between both groups. However, no big differences throughout the recordings, during night or day, are appreciable.

Table 2. SDNN calculated for the 24-hours recordings. Values shown are the inter-subject median and quartiles $\left(Q_{1}, Q_{3}\right)$.

\begin{tabular}{l|ll|l}
\hline \hline & S-group & F-group & $p$ \\
\hline SDNN $[\mathrm{ms}]$ & $57[46-108]$ & $38[30-58]$ & 0.07 \\
\hline \hline
\end{tabular}

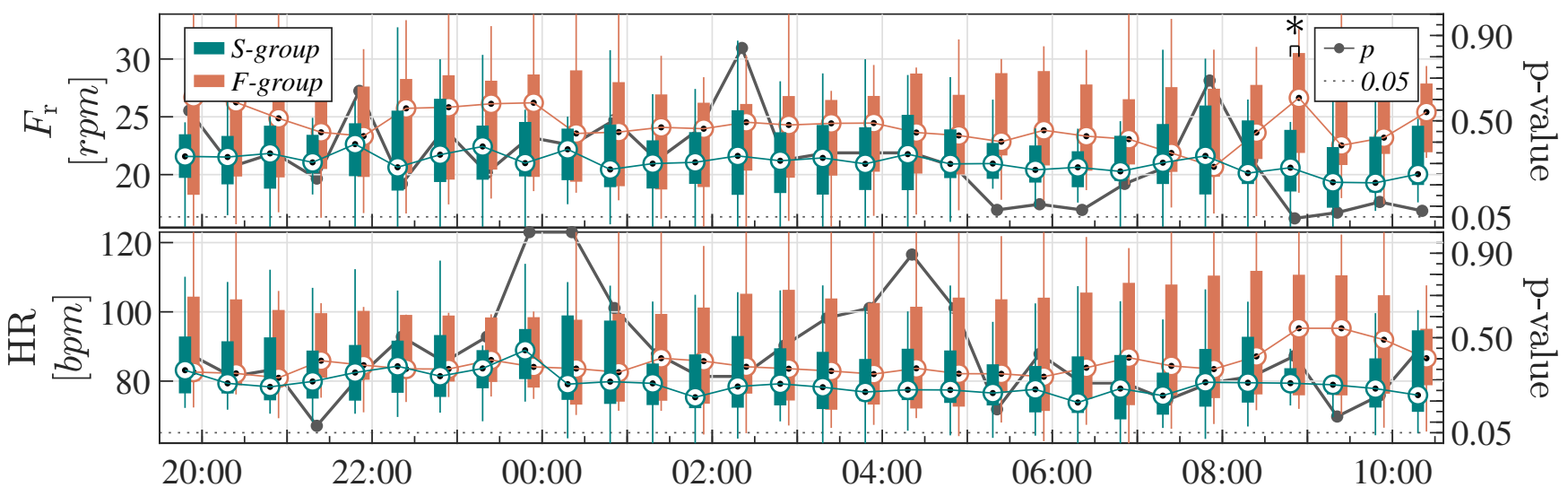

Figure 5. Evolution of the common clinical indices for weaning readiness before SBT. The mean respiratory frequency, $\mathrm{F}_{r}$, and mean HR, are represented. Green and Red boxplots represent the patients of the $S$-group and the $F$-group, respectively. The $p$-value comparing each half hour is represented in the right axis, from 0 to 1 , and the dotted line represents the $p=0.05$ threshold. The asterisks indicate statistical significance with $p \leq 0.05$.

Fig.6 shows the evolution of the HRV parameters. The RMSSD is higher in the S-group during the entire recording since midnight, apparently the moment when patients fall asleep. In particular, after waking up, at around 7:00 a.m., significant differences are found. Correspondingly, looking at the $\mathrm{P}_{\mathrm{LF}}^{n}$, an increment can be seen for the F-group, starting at 00:00, compared to the slight decrease for the $S$-group. This increment for the $F$-group can be associated with a SNS activation, in view of the sudden increase of the $\mathrm{P}_{\mathrm{HF}}$ and $\mathrm{P}_{\mathrm{VLF}}$ at the very same time. Curiously, sudden changes can sometimes be found on the $\mathrm{P}_{\mathrm{VLF}}$, especially for the $F$-group. However, much variability exists for the $\mathrm{P}_{\mathrm{VLF}}$ power, and neither significant differences nor appreciable patterns on the $\mathrm{P}_{\mathrm{VLF}}$ evolution can be found. 

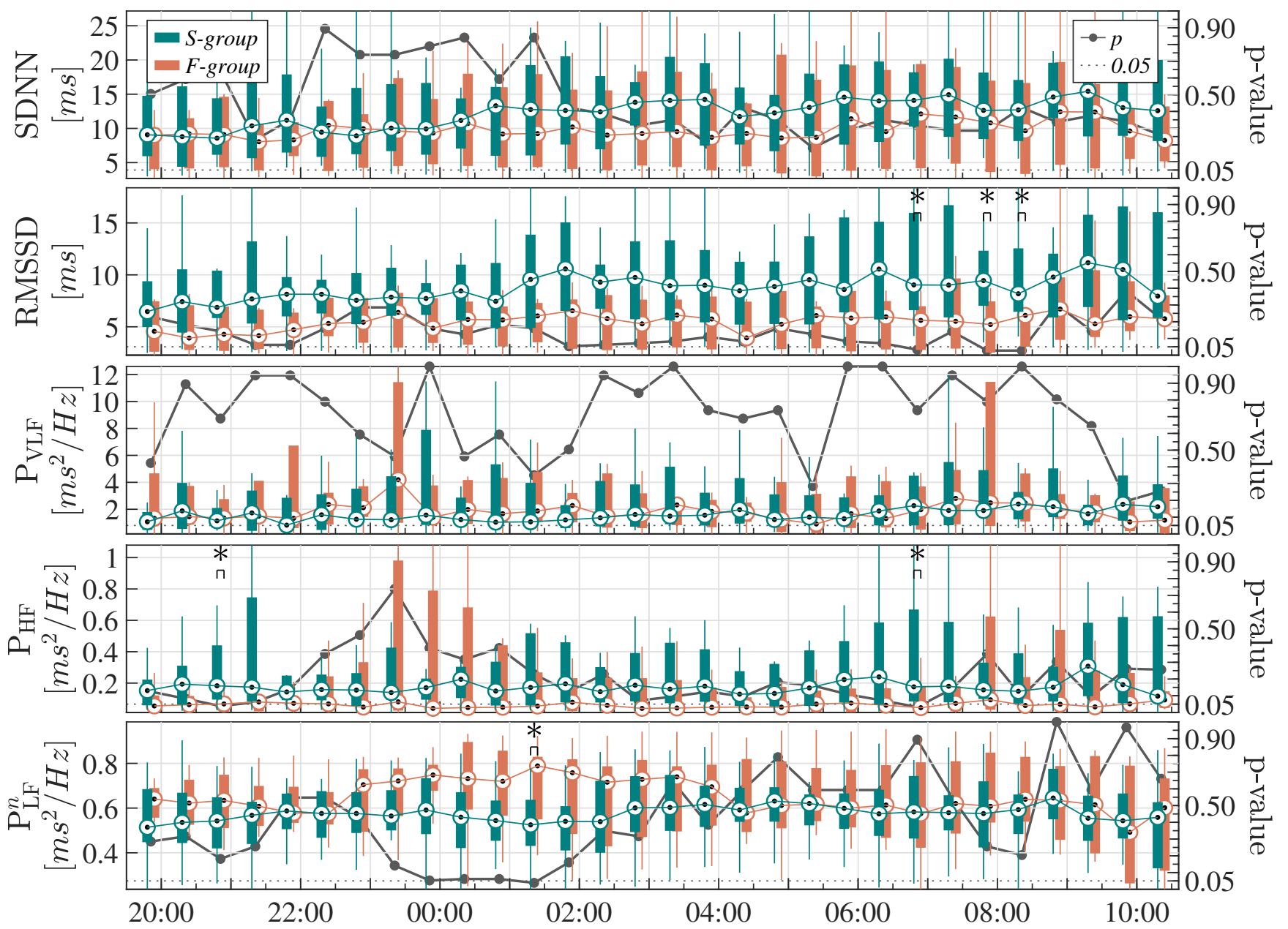

Figure 6. Evolution of the HRV indices before SBT. The temporal parameters SDNN and RMSSD, and the frequency parameters, $\mathrm{P}_{\mathrm{VLF}}, \mathrm{P}_{\mathrm{HF}}$ and $\mathrm{P}_{\mathrm{LF}}^{n}$ are represented. Green and Red boxplots represent the patients of the $S$-group and the F-group, respectively. The $p$-value comparing each half hour is represented in the right axis, from 0 to 1 , and the dotted line represents the $p=0.05$ threshold. The asterisks indicate statistical significance with $p \leq 0.05$.

The evolution of the CPC estimates is illustrated in Fig.7. As said, CPC estimators are computed considering respiration to be the system driving changes in the HRV. Clear differences exist in the CPC mechanism comparing the patients that were successfully weaned, $S$-group, and the patients reintubated or that still needed time in MV, F-group. The $\mathscr{C}_{\mathrm{HF}}^{\mathcal{T}}$ index quantifies the mean coherence normalized by the amount of time in coherence, in the HF band centred at the respiratory frequency. It is higher when HRV and respiration have components at the same frequencies, taking into account that these components have different physiological origin. Differences are significantly higher, particularly during the night. The $\mathscr{C} \mathscr{E}_{\mathrm{r} \leftrightarrow \mathrm{m}}$ term, that represents the amount of information shared between respiration and HRV, is larger for the S-group. This amount of information shared is higher during the night than in the morning before the SBT, especially for the $S$-group. The $\mathscr{P}_{\mathrm{m}_{\mathrm{r}}}$ represents the relative power of the respiratory component inserted within the HRV. It is also higher for the $S$-group than for the F-group. Remark that the $p$-values since 9:00 a.m. approximately, right before the SBT, increases abruptly for the 3 CPC indices. This means that the differences between the two groups are less substantial for the time before performing the SBT.

\section{Discussion}

HRV and CPC have been analysed for a total of 22 patients presumably ready for weaning, in the 24 hours before the SBT. Statistical differences have been found comparing patients who needed reintubation or required more time in MV, the so-called $F$-group, and patients with a successful extubation process, $S$-group. These differences are especially appreciable for the parameters estimating the CPC. The fact that the CPC changes so much with respect to the $S$-group can be related to a more unstable regulatory system. By monitoring this in the night, or in a continuous way, clinicians can obtain additional information 


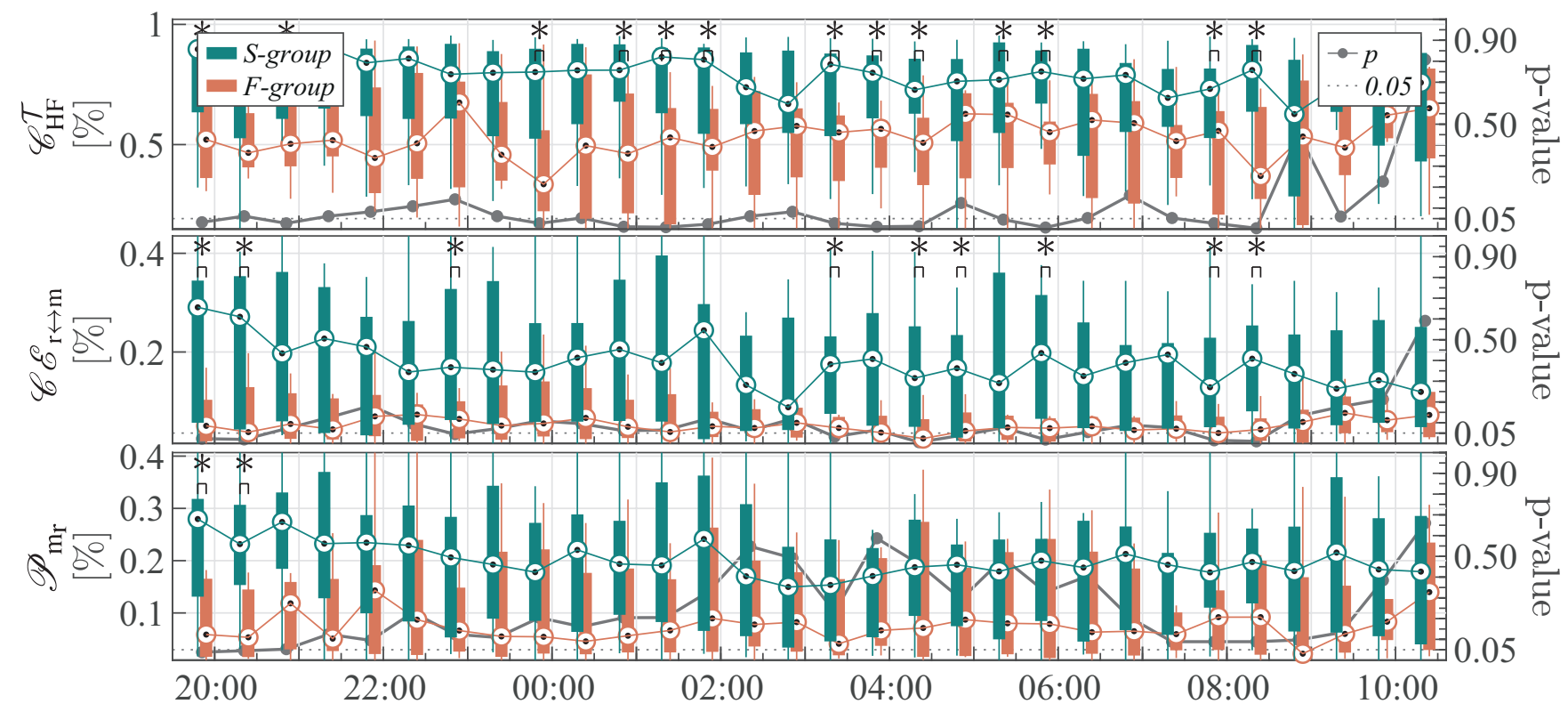

Figure 7. Evolution of the CPC estimators before SBT. The CPC parameters $\mathscr{C}_{\mathrm{HF}}^{\mathcal{T}}, \mathscr{C} \mathscr{E}_{\mathrm{r} \leftrightarrow \mathrm{m}}$ and $\mathscr{P}_{\mathrm{mr}}$, are represented. Green and Red boxplots represent the patients of the $S$-group and the $F$-group, respectively. The $p$-value comparing each half hour is represented in the right axis, from 0 to 1 , and the dotted line represents the $p=0.05$ threshold. The asterisks indicate statistical significance with $p \leq 0.05$.

of this stability that can help to make the decision to wean a patient from MV.

The patients of the $S$-group have higher values of SDNN, calculated over 24 hours (see Tab.2). A major component of SDNN is due to a higher variability and day-night difference of the HR. This shows a better adaptability of the heart to changes, for patients actually ready for weaning, $S$-group.

Remark that the SBT is not performed at the same time for all the patients. Hence, in order to have all recordings of the patients aligned in time, some segments had to be omitted at the start and end of the recordings for some patients.

The mean respiratory frequency, $\mathrm{F}_{r}$, was always above $9 \mathrm{rpm}$, i.e., $0.15 \mathrm{~Hz}$. However, for some patients, it was above 24 rpm, i.e., $0.4 \mathrm{~Hz}$ (see Fig.5). Moreover, in other studies like ${ }^{61}$, they found that HRV analysis guided by respiration improved the ability of HRV to discriminate cognitive stress in healthy subjects. Therefore, the need to centre the HF band in respiration is crucial to make a proper and more powerful interpretation of the results in the frequency domain.

The evolution of the currently-used clinical variables, $\mathrm{HR}$ and $\mathrm{F}_{r}$, is very similar for both groups. It is clear that these parameters are not giving useful information to predict weaning readiness. On the contrary, some HRV parameters seem to better discern both groups. The temporal parameter RMSSD has higher values for the S-group, in accordance with the fact that this index quantifies parasympathetic modulation of $\mathrm{NN}$ intervals driven by respiration and vagal modulations ${ }^{60}$.

These modulations of the vagal activity are also quantified by $\mathrm{P}_{\mathrm{HF}}$. Notice that the $\mathrm{P}_{\mathrm{HF}}$ is much higher for some patients of the F-group, around 11 p.m., and around 8:00 a.m. The rest of the time, mainly during sleep at night, $\mathrm{P}_{\mathrm{HF}}$ is higher for the $S$-group, in agreement with the results of ${ }^{28}$. The time when $\mathrm{P}_{\mathrm{HF}}$ is higher for the $F$-group, occurs before going to sleep and waking up. However, the $\mathrm{P}_{\mathrm{VLF}}$ and $\mathrm{P}_{\mathrm{LF}}^{n}$ are also higher, so strong vagal modulations are in conflict with strong sympathetic activations. Moreover, this sudden increase in the $\mathrm{P}_{\mathrm{HF}}$ for the $F$-group, that could be interpreted as an increase of the vagal activity, is not present in the CPC parameters. These patients are an example where the frequency content in the HF band does not contain only respiratory information (see Fig.4). These HF components can be a consequence of the non-linear effects of respiration transferred to the HR. These non-linear influences could be mediated by the respiratory pacemaker in the central nervous system ${ }^{62}$ through SNS modulations ${ }^{63}$, and the CPC estimates used in this work are unable to detect them. Further investigation is required using techniques able to take both linear and non-linear effects into account. Methodologies that are able to quantify up to second order interactions by detecting and quantifying the quadratic phase coupling ${ }^{64}$ could account for this fact or also techniques based on least-squares support vector machines formulated for non-linear function estimation ${ }^{65}$.

The $\mathrm{P}_{\mathrm{LF}}^{n}$ is the standard measure of the sympathovagal balance. However, it has already been proven that $\mathrm{P}_{\mathrm{LF}}^{n}$ is not an appropriate measure of the vagal and sympathetic modulations ${ }^{46,66}$, and that maybe is why only slight differences can be appreciated during the night. Therefore, in this context of the ICU, where patients are continuously monitored, $\mathrm{P}_{\mathrm{LF}}^{n}$ is not 
recommended as an index to use as a reliable weaning predictor.

At this point, results of the CPC parameters were encouraging. First, the TFC exhibited illustrative results. The $\mathscr{C}_{\mathrm{HF}}^{\mathcal{T}}$, is the CPC estimator which exhibits larger differences between $S$-group and $F$-group patients. These results are also in agreement with those obtained using mutual information, where higher $\mathscr{C} \mathscr{E}_{\mathrm{r} \leftrightarrow \mathrm{m}}$ values were found in the S-group than in the F-group. Finally, there are also visible differences looking at the relative power of respiration, $\mathscr{P}_{\mathrm{m}_{\mathrm{r}}}$, inserted into the HRV: patients of the $S$-group had a relative power around the $25 \%$ of respiration, but those of the $F$-group had it around 5\%. Altogether, this illustrates that those patients who are actually ready for weaning, have good levels of RSA and that their CPC system is ready to work, in contrast with the patients who did not pass SBT or needed reintubation. Therefore, these CPC estimators are promising as additional indexes to improve the weaning readiness criteria.

Moreover, these differences in the CPC parameters are more evident during sleep than right before the SBT, what could be due to the loop gain. In other words, patients with failed weaning might be experiencing more apnoea events during the night, which is directly related to a reduced RSA and a higher cardiovascular risk ${ }^{40,67}$. In fact, the PNS activity is well known to be predominant during sleep at night. Consequently, it is in this moment when the CPC is stronger. Results suggest that the proper moment to test readiness is during the night, more than during the early morning. Probably, it would be better to check at night how the patient behaves to decide whether to wean. However, one limitation is that the patients in the ICU may not keep similar sleep patterns. Nowadays, clinicians assess whether the patients can perform SBT when they are awake in the morning and conscious. The patients, knowing that they have to prepare for the SBT, can generate high levels of stress and anxiety, and this can alter their biomarkers (HR, $\mathrm{F}_{r}, \mathrm{CPC}$ estimates, $\mathrm{SpO}_{2}, \mathrm{HRV}$, etc.), towards more alert-related values. In point of fact, less clear differences are found at this time, in the morning right before the SBT.

The computational cost for obtaining $\mathscr{P}_{\mathrm{m}_{\mathrm{r}}}$ is low and it may be obtained in real time in the same way as the standard clinical parameters. On the other hand, the computational cost for obtaining $\mathscr{C} \mathscr{E} \mathrm{r \leftrightarrow m}$ or $\mathscr{C}_{\mathrm{HF}}^{\mathcal{T}}$ is higher, but they could also be obtained in quasi-real time. Therefore, these algorithms can be implemented in the ICU monitors, and the CPC status could be assessed continuously, together with the well-known clinical variables.

In a preliminary analysis of this study, other CPC estimators were also explored, like the other entropy measures from the ID framework, the bandwidth of the TFC and the unconstrained sympathovagal balance from the HRV decomposition. However, the indices with best performance were the ones presented here, namely $\mathscr{P}_{\mathrm{mr}}, \mathscr{C} \mathscr{E}_{\mathrm{r} \leftrightarrow \mathrm{m}}$ and $\mathscr{C}_{\mathrm{HF}}^{\mathcal{T}}$. Interestingly, the study in ${ }^{50}$ analyses the best methods for RSA estimation in a simulation study, and concludes that the same three parameters are the best estimators of the CPC.

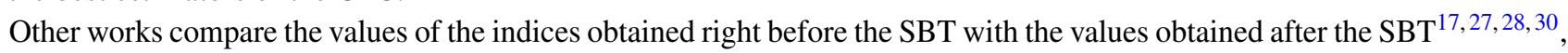
and all of them found some differences in the HRV parameters. The very important difference of the present study with the state-of-the-art is that the indices and evolution of the patients are obtained only before the SBT and using the long-term information. The fact that the weaning readiness prediction can be improved using CPC indices could only have been revealed using long-term analysis.

$\mathrm{In}^{68}$, they stress the importance of the $\mathrm{P}_{\mathrm{VLF}}$ power in the weaning scenario. The $\mathrm{P}_{\mathrm{VLF}}$ power, partially related with the circadian rhythms ${ }^{60}$, could provide useful information of the neurohumoral regulatory mechanism ${ }^{69}$. On the contrary, here it is illustrated that the $\mathrm{P}_{\mathrm{VLF}}$ power is not so relevant in the analysis of the 24 hours prior to SBT. Nevertheless, some patients in this MV and ICU context showed strong characteristic VLF oscillations, possibly also related with sleep disorders, that must be further studied. These sudden changes cause non-stationarity, and this is the reason why 3-minute sliding windows and TF analysis are used to calculate the indices.

Further studies should be performed including controlled MV modes, in order to obtain a complete knowledge of the CPC regulation mechanisms during the whole weaning process. As said, only patients in CPAP or PSV modes are analysed in this study as these MV modes force spontaneous breathing. Therefore, there is no influence of the ventilator into the CPC mechanisms, through automatized respiration driven by the ventilator. Perhaps, in the controlled MV modes, it is the ventilator the one controlling respiration -not the ANS-, and the HRV would be the driving system, since the ventilator would be the one regulating respiration externally. This might lead to a different impact on the CPC estimators, since synchronization and coordination of the regulatory mechanism could change.

The clinical utility of this, and future studies, is that if HRV and CPC are actually proven to predict weaning failure, they might be incorporated as screening parameters. In other words, HRV and CPC could be calculated or assessed before weaning, in a multimodal index combined with the current parameters to reinforce the prediction of weaning success or failure. In fact, looking at Tab.1, patients with failed weaning had a higher mortality rate, and spent more days in MV and in the ICU. Therefore, this multimodal index could help to reduce these weaning failure rates and the very adverse effects associated with reintubation, to thereby result in better clinical outcomes.

Finally, it must be kept in mind that patients in the ICU are admitted from very diverse diagnostics. Taking this into account, two patients with the same characteristics and similar evolution may get different outcomes. Hence, sometimes, a patient who does not meet the readiness criteria can be also successfully weaned, and viceversa ${ }^{15}$. This is why clinicians take the criteria for 
weaning readiness and SBT performance as one among several considerations rather than rigid requirements. Such uncertainty can be reduced by implementing new research and technologies to daily clinical practice ${ }^{70,71}$, and this study is other step forward in the field of predictive precision medicine, that exploits the capabilities of the CPC estimates.

\section{Conclusions}

This study analysed the evolution of patients undergoing weaning from MV in the 24 hours before the SBT, considering that all patients were "ready" for discontinuation from MV. Furthermore, this evaluation was done looking only at the results before performing the SBT, but because of this, it was needed a long-term analysis.

None of the parametric measures currently used as clinical criteria for weaning readiness showed noticeable differences between the patients actually ready for weaning and the patients who did not pass the SBT or those who needed reintubation. However, it was revealed that patients successfully weaned exhibited higher CPC values, assessed with the variables $\mathscr{C}_{\mathrm{HF}}^{\mathcal{T}}$, $\mathscr{C} \mathscr{E}_{\mathrm{r} \leftrightarrow \mathrm{m}}$ and $\mathscr{P}_{\mathrm{m}}$, specially, during the night prior to the SBT. In addition, it was shown that some differences exist for the HRV parameters, but not as strong as for the CPC indices.

As it was stated, the information that clinicians are handling in the ICU at this moment is not enough to decide weaning readiness. By way of conclusion, it was found that these CPC indices have information of the weaning readiness, but even before performing the SBT. The potential predictive value of these CPC indices should be considered and further studied, in order to implement this technology into the daily clinical practice, integrating software systems like Better Care in the ICU's. This will help to reduce weaning failure rates, the very adverse effects associated with the reintubation process and thereby result in better clinical outcomes.

\section{References}

1. Ouellette, D. R. et al. Liberation from mechanical ventilation in critically ill adults: an official american college of chest physicians/american thoracic society clinical practice guideline: inspiratory pressure augmentation during spontaneous breathing trials, protocols minimizing sedation, and noninvasive ventilation immediately after extubation. Chest 151, 166-180 (2017).

2. Boles, J.-M. et al. Weaning from mechanical ventilation. Eur. Respir. J. 29, 1033-1056 (2007).

3. Epstein, S. K., Ciubotaru, R. L. \& Wong, J. B. Effect of failed extubation on the outcome of mechanical ventilation. Chest 112, 186-192 (1997).

4. Thille, A. W., Harrois, A., Schortgen, F., Brun-Buisson, C. \& Brochard, L. Outcomes of extubation failure in medical intensive care unit patients. Critical care medicine 39, 2612-2618 (2011).

5. Torres, A. et al. Re-intubation increases the risk of nosocomial pneumonia in patients needing mechanical ventilation. Am. journal respiratory critical care medicine 152, 137-141 (1995).

6. Esteban, A. et al. Extubation outcome after spontaneous breathing trials with t-tube or pressure support ventilation. Am. journal respiratory critical care medicine 156, 459-465 (1997).

7. Esteban, A. et al. Effect of spontaneous breathing trial duration on outcome of attempts to discontinue mechanical ventilation. Am. journal respiratory critical care medicine 159, 512-518 (1999).

8. Vallverdu, I. et al. Clinical characteristics, respiratory functional parameters, and outcome of a two-hour t-piece trial in patients weaning from mechanical ventilation. Am. journal respiratory critical care medicine 158, 1855-1862 (1998).

9. Frutos-Vivar, F. et al. Outcome of reintubated patients after scheduled extubation. J. critical care 26, 502-509 (2011).

10. Jubran, A. \& Tobin, M. J. Pathophysiologic basis of acute respiratory distress in patients who fail a trial of weaning from mechanical ventilation. Am. journal respiratory critical care medicine 155, 906-915 (1997).

11. Tobin, M. J. et al. Konno-mead analysis of ribcage-abdominal motion during successful and unsuccessful trials of weaning from mechanical ventilation. Am. Rev. Respir. Dis. 135, 1320-1328 (1987).

12. Slutsky, A. S. \& Ranieri, V. M. Ventilator-induced lung injury. New Engl. J. Medicine 369, $2126-2136$ (2013).

13. Thille, A. W., Richard, J.-C. M. \& Brochard, L. The decision to extubate in the intensive care unit. Am. journal respiratory critical care medicine 187, 1294-1302 (2013).

14. Navalesi, P. et al. Prolonged weaning: from the intensive care unit to home. Revista portuguesa de pneumologia 20, 264-272 (2014).

15. Ely, E., Baker, A., Evans, G. \& Haponik, E. The prognostic significance of passing a daily screen of weaning parameters. Intensive care medicine 25, 581-587 (1999). 
16. Meade, M. et al. Predicting success in weaning from mechanical ventilation. Chest 120, 400S-424S (2001).

17. Huang, C.-T. et al. Application of heart-rate variability in patients undergoing weaning from mechanical ventilation. Critical care 18, R21 (2014).

18. Papaioannou, V. E., Chouvarda, I. G., Maglaveras, N. K. \& Pneumatikos, I. A. Study of multiparameter respiratory pattern complexity in surgical critically ill patients during weaning trials. BMC physiology 11, 2 (2011).

19. Zein, H., Baratloo, A., Negida, A. \& Safari, S. Ventilator weaning and spontaneous breathing trials; an educational review. Emergency 4, 65 (2016).

20. MacIntyre, N. R. et al. Evidence-based guidelines for weaning and discontinuing ventilatory support. Chest 120, 375S-95S (2001).

21. Esteban, A. et al. Evolution of mechanical ventilation in response to clinical research. Am. journal respiratory critical care medicine 177, 170-177 (2008).

22. Thomas, G. D. Neural control of the circulation. Adv. physiology education 35, 28-32 (2011).

23. Malpas, S. C. Sympathetic nervous system overactivity and its role in the development of cardiovascular disease. Physiol. reviews 90, 513-557 (2010).

24. Schmidt, H. et al. The alteration of autonomic function in multiple organ dysfunction syndrome. Critical care clinics $\mathbf{2 4}$, 149-163 (2008).

25. Wieske, L. et al. Autonomic dysfunction in icu-acquired weakness: a prospective observational pilot study. Intensive care medicine 39, 1610-1617 (2013).

26. Ryan, M. L. et al. Heart rate variability is an independent predictor of morbidity and mortality in hemodynamically stable trauma patients. J. Trauma Acute Care Surg. 70, 1371-1380 (2011).

27. Shen, H.-N. et al. Changes of heart rate variability during ventilator weaning. Chest 123, 1222-1228 (2003).

28. Chen, Y.-J. et al. Vagal withdrawal and psychological distress during ventilator weaning and the related outcomes. $J$. Psychosom. Res. 101, 10-16 (2017).

29. Seely, A. J. et al. Do heart and respiratory rate variability improve prediction of extubation outcomes in critically ill patients? Critical Care 18, R65 (2014).

30. Arcentales, A., Caminal, P., Diaz, I., Benito, S. \& Giraldo, B. Classification of patients undergoing weaning from mechanical ventilation using the coherence between heart rate variability and respiratory flow signal. Physiol. measurement 36, 1439 (2015).

31. Hammash, M. H., Moser, D. K., Frazier, S. K., Lennie, T. A. \& Hardin-Pierce, M. Heart rate variability as a predictor of cardiac dysrhythmias during weaning from mechanical ventilation. Am. J. Critical Care 24, 118-127 (2015).

32. Berntson, G. G., Cacioppo, J. T. \& Quigley, K. S. Respiratory sinus arrhythmia: autonomic origins, physiological mechanisms, and psychophysiological implications. Psychophysiology 30, 183-196 (1993).

33. Larsen, P., Tzeng, Y., Sin, P. \& Galletly, D. Respiratory sinus arrhythmia in conscious humans during spontaneous respiration. Respir. physiology \& neurobiology 174, 111-118 (2010).

34. Yasuma, F. \& Hayano, J.-i. Respiratory sinus arrhythmia: why does the heartbeat synchronize with respiratory rhythm? Chest 125, 683-690 (2004).

35. Dick, T. E. et al. Cardiorespiratory coupling: common rhythms in cardiac, sympathetic, and respiratory activities. In Progress in brain research, vol. 209, 191-205 (Elsevier, 2014).

36. Eckberg, D. L. Point: counterpoint: respiratory sinus arrhythmia is due to a central mechanism vs. respiratory sinus arrhythmia is due to the baroreflex mechanism. J. applied physiology (2009).

37. Karemaker, J. M. Counterpoint: respiratory sinus arrhythmia is due to the baroreflex mechanism. J. applied physiology 106, 1742-1743 (2009).

38. Julien, C. et al. Comments on point: counterpoint: respiratory sinus arrhythmia is due to a central mechanism vs. respiratory sinus arrhythmia is due to the baroreflex mechanism. J. Appl. Physiol. 106, 1745-1749 (2009).

39. Thomas, R. J., Wood, C. \& Bianchi, M. T. Cardiopulmonary coupling spectrogram as an ambulatory clinical biomarker of sleep stability and quality in health, sleep apnea, and insomnia. Sleep 41, zsx196 (2018).

40. Varon, C., Caicedo, A., Testelmans, D., Buyse, B. \& Van Huffel, S. A novel algorithm for the automatic detection of sleep apnea from single-lead ecg. IEEE Transactions on Biomed. Eng. 62, 2269-2278 (2015). 
41. Sun, Q.-M., Xing, L., Wang, C. \& Liang, W. Cardiopulmonary coupling analysis predicts early treatment response in depressed patients: A pilot study. Psychiatry research 276, 6-11 (2019).

42. Milagro, J. et al. Noninvasive cardiorespiratory signals analysis for asthma evolution monitoring in preschool children. IEEE Transactions on Biomed. Eng. (2019).

43. Electrophysiology, T. F. o. t. E. S. o. C. t. N. A. S. o. P. Heart rate variability: standards of measurement, physiological interpretation, and clinical use. Circulation 93, 1043-1065 (1996).

44. Orini, M., Bailón, R., Mainardi, L. T., Laguna, P. \& Flandrin, P. Characterization of dynamic interactions between cardiovascular signals by time-frequency coherence. IEEE transactions on biomedical engineering 59, 663-673 (2011).

45. Faes, L., Porta, A., Nollo, G. \& Javorka, M. Information decomposition in multivariate systems: definitions, implementation and application to cardiovascular networks. Entropy 19, 5 (2017).

46. Varon, C. et al. Unconstrained estimation of hrv indices after removing respiratory influences from heart rate. IEEE journal biomedical health informatics 23, 2386-2397 (2018).

47. Schumann, A. Y., Kantelhardt, J. W., Bauer, A. \& Schmidt, G. Bivariate phase-rectified signal averaging. Phys. A: Stat. Mech. its Appl. 387, 5091-5100 (2008).

48. Faes, L., Krohova, J., Pernice, R., Busacca, A. \& Javorka, M. A new frequency domain measure of causality based on partial spectral decomposition of autoregressive processes and its application to cardiovascular interactions. In $201941 s t$ Annual International Conference of the IEEE Engineering in Medicine and Biology Society (EMBC), 4258-4261 (IEEE, 2019).

49. Bartsch, R. P., Schumann, A. Y., Kantelhardt, J. W., Penzel, T. \& Ivanov, P. C. Phase transitions in physiologic coupling. Proc. Natl. Acad. Sci. 109, 10181-10186 (2012).

50. Morales, J. et al. Model-based evaluation of methods for respiratory sinus arrhythmia estimation. IEEE transactions on biomedical engineering ?, ? (?).

51. Blanch, L. et al. Validation of the better care ${ }^{\circledR}$ system to detect ineffective efforts during expiration in mechanically ventilated patients: a pilot study. Intensive care medicine 38, 772-780 (2012).

52. de Haro, C. et al. Double cycling during mechanical ventilation: frequency, mechanisms, and physiologic implications. Critical care medicine 46, 1385-1392 (2018).

53. Merri, M., Farden, D. C., Mottley, J. G. \& Titlebaum, E. L. Sampling frequency of the electrocardiogram for spectral analysis of the heart rate variability. IEEE Transactions on Biomed. Eng. 37, 99-106 (1990).

54. Sörnmo, L. \& Laguna, P. Bioelectrical signal processing in cardiac and neurological applications, vol. 8 (Academic Press, 2005).

55. Martínez, J. P., Almeida, R., Olmos, S., Rocha, A. P. \& Laguna, P. A wavelet-based ecg delineator: evaluation on standard databases. IEEE Transactions on biomedical engineering 51, 570-581 (2004).

56. Mateo, J. \& Laguna, P. Analysis of heart rate variability in the presence of ectopic beats using the heart timing signal. IEEE Transactions on biomedical engineering 50, 334-343 (2003).

57. Bailón, R. et al. The integral pulse frequency modulation model with time-varying threshold: application to heart rate variability analysis during exercise stress testing. IEEE transactions on biomedical engineering 58, 642-652 (2010).

58. Bailón, R., Laguna, P., Mainardi, L. \& Sornmo, L. Analysis of heart rate variability using time-varying frequency bands based on respiratory frequency. In 2007 29th Annual International Conference of the IEEE Engineering in Medicine and Biology Society, 6674-6677 (IEEE, 2007).

59. Pagani, M. et al. Power spectral analysis of heart rate and arterial pressure variabilities as a marker of sympatho-vagal interaction in man and conscious dog. Circ. research 59, 178-193 (1986).

60. Shaffer, F. \& Ginsberg, J. An overview of heart rate variability metrics and norms. Front. public health 5, 258 (2017).

61. Hernando, A. et al. Inclusion of respiratory frequency information in heart rate variability analysis for stress assessment. IEEE journal biomedical health informatics 20, 1016-1025 (2016).

62. Peña, F. Contribution of pacemaker neurons to respiratory rhythms generation in vitro. In Integration in Respiratory Control, 114-118 (Springer, 2008).

63. Saul, J. P. et al. Transfer function analysis of the circulation: unique insights into cardiovascular regulation. Am. J. Physiol. Circ. Physiol. 261, H1231-H1245 (1991). 
64. Kontaxis, S., Lázaro, J., Gil, E., Laguna, P. \& Bailón, R. Assessment of quadratic nonlinear cardiorespiratory couplings during tilt-table test by means of real wavelet biphase. IEEE Transactions on Biomed. Eng. 66, 187-198 (2018).

65. Varon, C., Hendrikx, D., Bolea, J., Laguna, P. \& Bailón, R. Quantification of linear and nonlinear cardiorespiratory interactions under autonomic nervous system blockade. In 2019 Computing in Cardiology (CinC), Page-1 (IEEE, 2019).

66. Billman, G. E. The lf/hf ratio does not accurately measure cardiac sympatho-vagal balance. Front. physiology 4, 26 (2013).

67. Milagro, J. et al. Autonomic dysfunction increases cardiovascular risk in the presence of sleep apnea. Front. Physiol. 10, 620 (2019).

68. Krasteva, V., Matveev, M., Jekova, I. \& Georgiev, G. Heart rate variability analysis during weaning from mechanical ventilation: Models for prediction of the weaning trial outcome. In 2018 Computing in Cardiology Conference (CinC), vol. 45, 1-4 (IEEE, 2018).

69. Frazier, S. K. et al. Autonomic tone in medical intensive care patients receiving mechanical ventilation and during a CPAP weaning trial. Biol. Res. for Nurs. 9, 301-310 (2008).

70. Marchuk, Y. et al. Predicting patient-ventilator asynchronies with hidden markov models. Sci. reports 8, 1-7 (2018).

71. Sarlabous, L. et al. Development and validation of a sample entropy-based method to identify complex patient-ventilator interactions during mechanical ventilation. Sci. reports 10, 1-12 (2020).

\section{Acknowledgements}

This work was supported by CIBER in Bioengineering, Biomaterials \& Nanomedicine and CIBER in Respiratory Diseases through Instituto de Salud Carlos III: INTERCIBER-PAI2017 ; by Ministerio de Economía y Competitividad (RTI2018-097723B-I00), Gobierno de Aragón (Reference Group BSICoS T39-20R) cofunded by the Fondo Europeo de Desarrollo Regional (FEDER) 2014-2020 "Building Europe from Aragón"; by a personal grant to P. Armañac, Programa Ibercaja-CAI de Estancias de Investigación, code IT 9/19; by PI16/01606, PI19/01015, integrated in the Plan Nacional de R+D+I and co-funded by the ISCIII- Subdirección General de Evaluación and the FEDER; RTC-2017-6193-1 (AEI/FEDER UE); and by Fundació Parc Taulí. This study has also received funding from the European Union's Framework Programme for Research and Innovation Horizon 2020 (2014-2020) under the Marie Skłodowska-Curie Grant Agreement No. 745755.

\section{Author contributions statement}

All authors contributed to the study meaningfully, providing feedback on the interpretation of the results and on the writing of the paper. P.AJ. conducted the study, analysis, and implementation of the methodologies. P.AJ., J.Mor., J.Moe., D.H., J.L., M.O. and C.V. implemented the algorithms for HRV analysis and CPC estimation. R.M. and L.S. registered, exported and checked the data for analysis. C.dH., J.LA., L.B., C.S. and R.F. provided the feedback and clinical vision during the analysis. C.dH., J.LA., P.L., C.V., E.G., R.B. and L.B. supervised the study. P.AJ. wrote the original draft manuscript.

\section{Competing interests}

L.B., C.dH., L.S., J.LA. and R.M. have been named in a provisional European patent application number EP19383116 owned by Corporació Sanitària Parc Taulí: "A device and method for respiratory monitoring in mechanically ventilated patients".

L.B. is inventor of a US patent owned by Corporació Sanitària Parc Taulí: "Method and system for managed related patient parameters provided by a monitoring device", US Patent No. 12/538,940.

L.B. owns stock options of BetterCare S.L., a research and development spin-off of Corporació Sanitària Parc Taulí.

The other authors P.AJ., D.H., J.L., J.Mor., J.Moe., C.S., R.F., M.O., P.L, C.V., E.G. and R.B. declare no competing interests. 

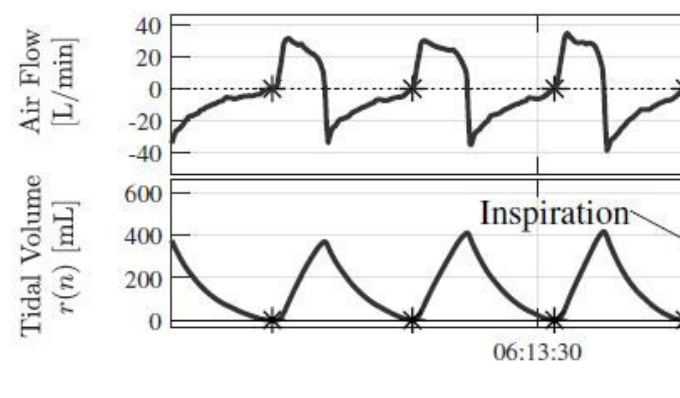
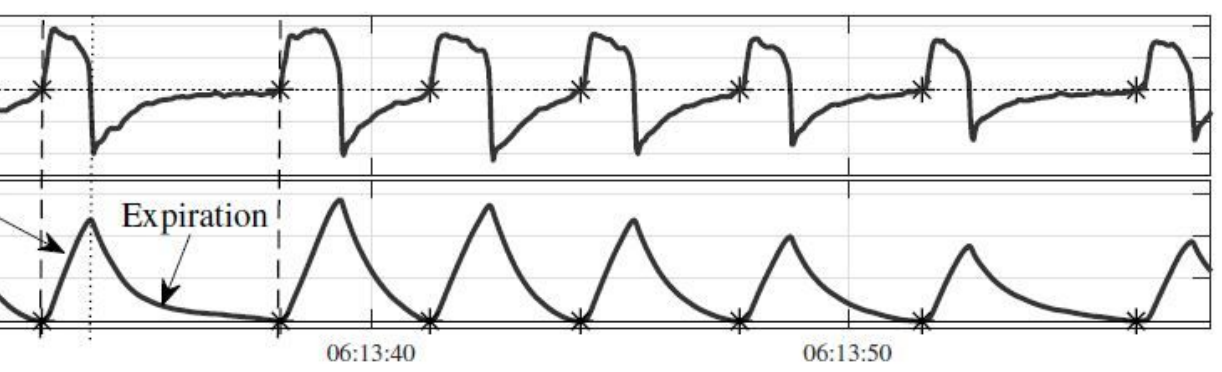

06:13:40

Time [HH:MM:SS]

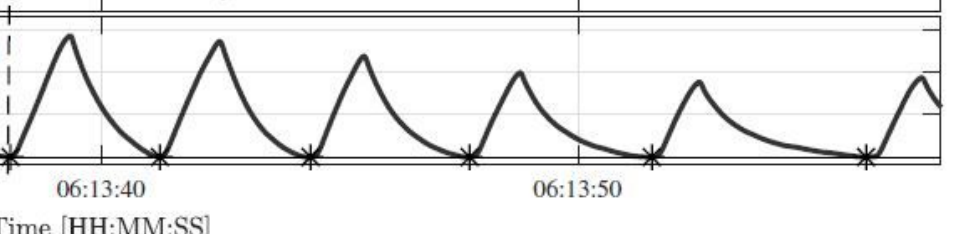

\section{Figure 1}

Example of respiration in PSV mode. The airflow signal is plotted at the (top) and the derived tidal volume signal at the (bottom). The onset of inspiration, $\mathrm{nl}, \mathrm{ON}$, is marked with asterisks.

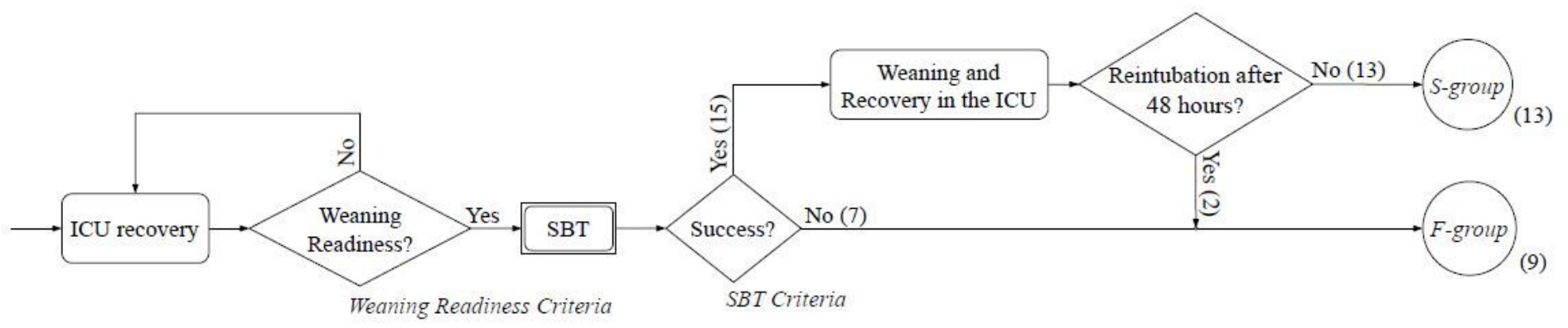

\section{Figure 2}

Algorithm for the definition of the weaning success. Patients are classified into the S-group or F-group after the Spontaneous Breathing Trial (SBT). The S-group stands for the group of patients successfully weaned (successful SBT and no need of reintubation). F-group stands for the group of patients with SBT failure and patients with SBT success but with the need of reintubation after 48 hours of weaning. Numbers in parentheses represent the number of patients according to the respective criteria. 


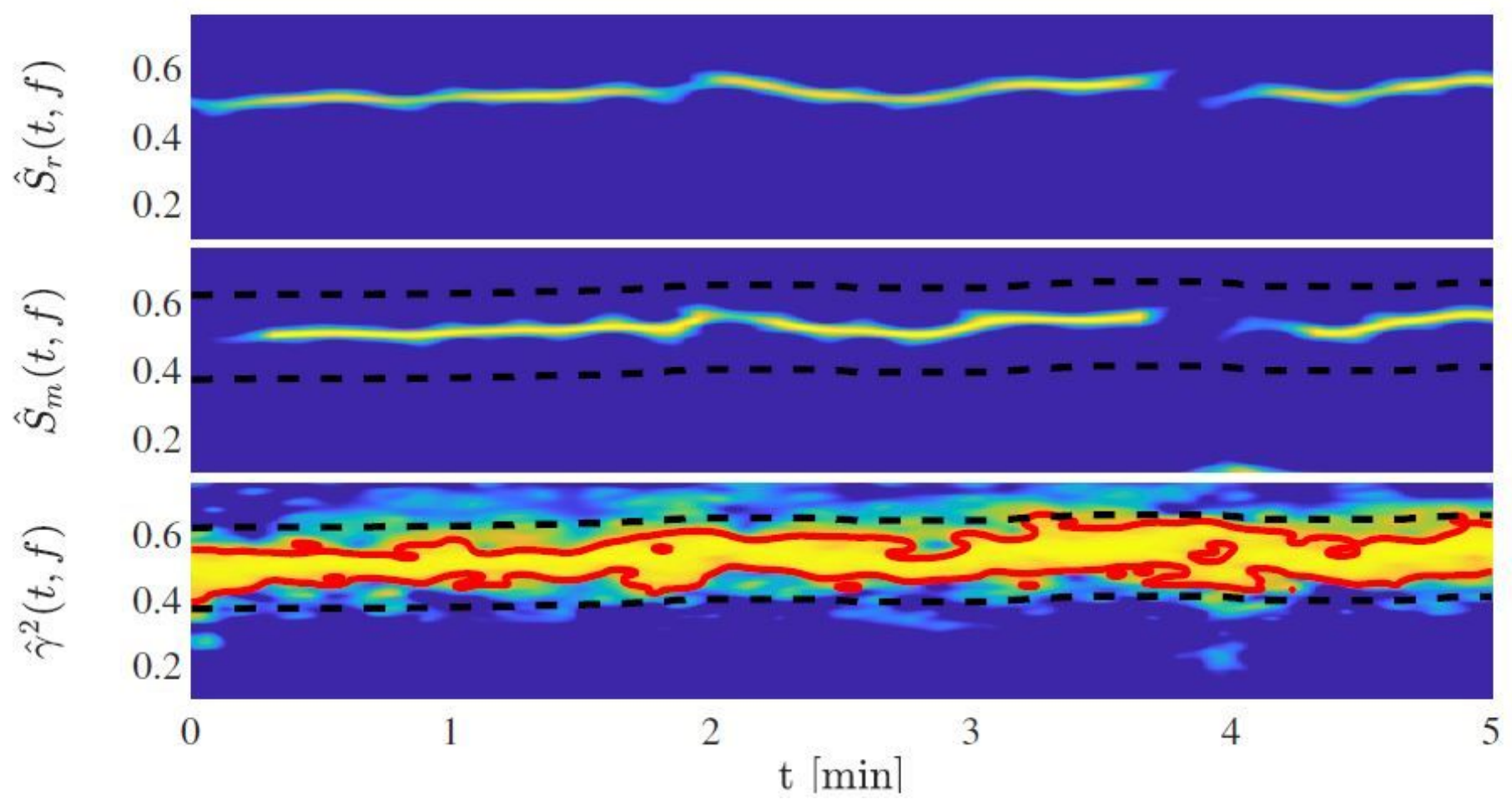

\section{Figure 3}

Time-frequency maps of HRV, respiration and spectral coherence of a five minutes period. The TF map of the respiratory signal, $S^{\wedge} r(t ; f)$, is on (top) and the TF map of the HRV modulating signal, $S^{\wedge} m(t ; f)$, is in the (middle). The TF map of the quadratic spectral coherence between respiration and HRV, ${ }^{\wedge} \gamma 2(t ; f)$, is in the (bottom). The white-dotted lines in the TF map of $S^{\wedge} m(t ; f)$ and $y^{\wedge} 2(t ; f)$, represent the HF band centred at the respiratory frequency, $\Omega \mathrm{rHF}(\mathrm{t})$. The regions of coherence with statistical significance within the $\mathrm{HF}$ band centred at the $\mathrm{Fr}, \Omega \mathrm{r} ; \mathrm{c} \mathrm{HF}(\mathrm{t} ; \mathrm{f})$, are the red-colored area in the ${ }^{\wedge} \mathrm{\gamma} 2(\mathrm{t} ; \mathrm{f})$ map. For further information, see44. 

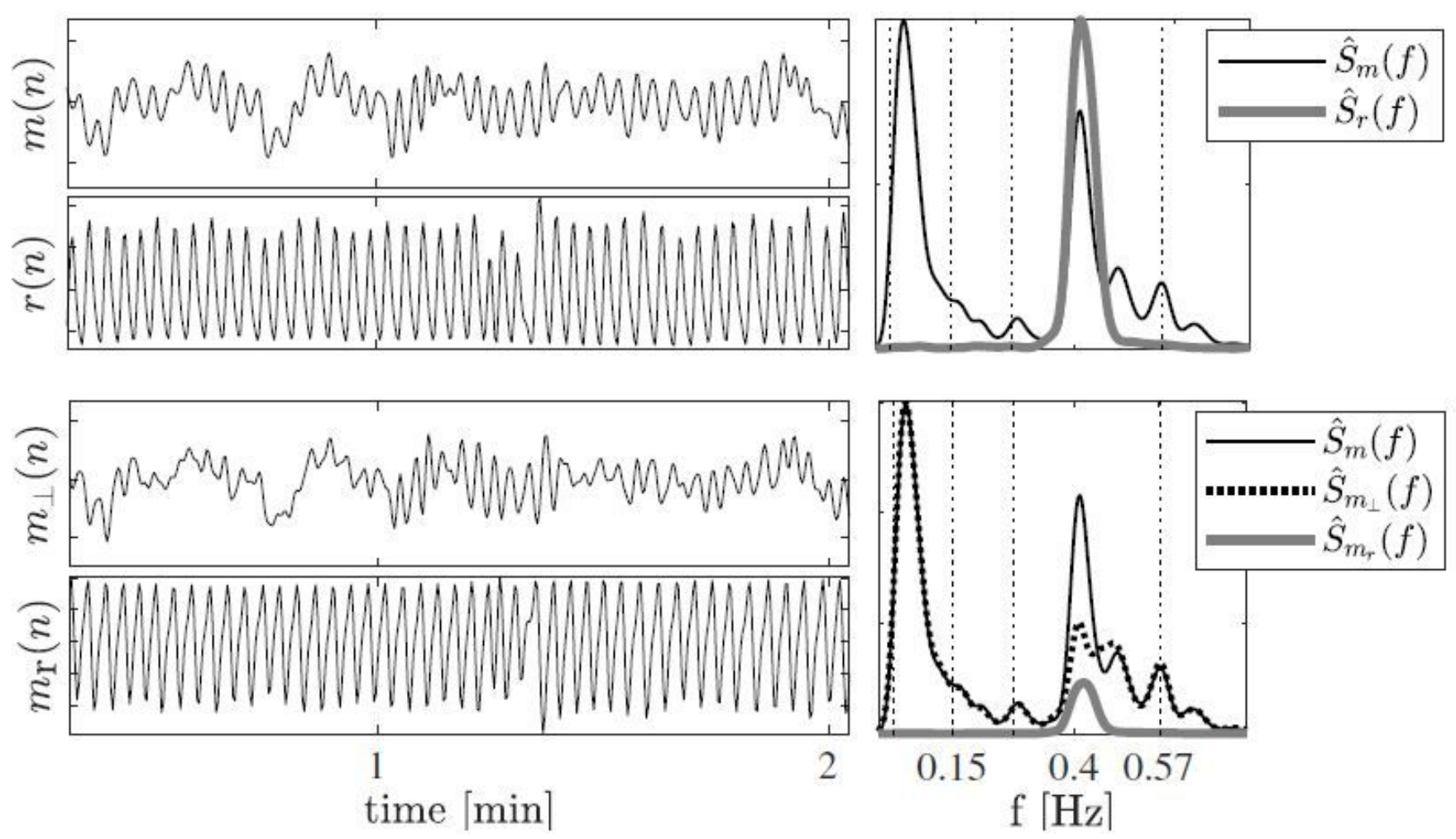

Figure 4

Illustrative example of the OSP decomposition using the HRV and Respiratory signals. The three upper plots are the time evolution of the modulating signal, $m(n)$, the respiratory signal, $r(n)$, and their respective spectra on the upper-right side, $S^{\wedge} m(f)$ for $m(n)$ and $S^{\wedge} r(f)$ for $r(n)$. The three plots below represent the OSP decomposition. The respiratory component of $\mathrm{HRV}, \mathrm{mr}(\mathrm{n})$, is obtained projecting $\mathrm{m}(\mathrm{n})$ onto the

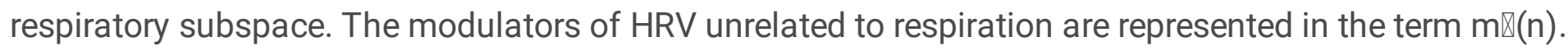

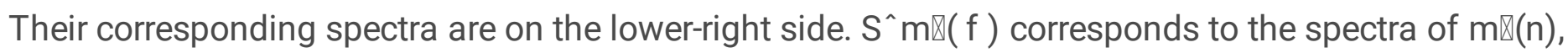
and $S^{\wedge} \mathrm{mr}(f)$ to the spectra of $\mathrm{mr}(\mathrm{n})$. For further information, see46.

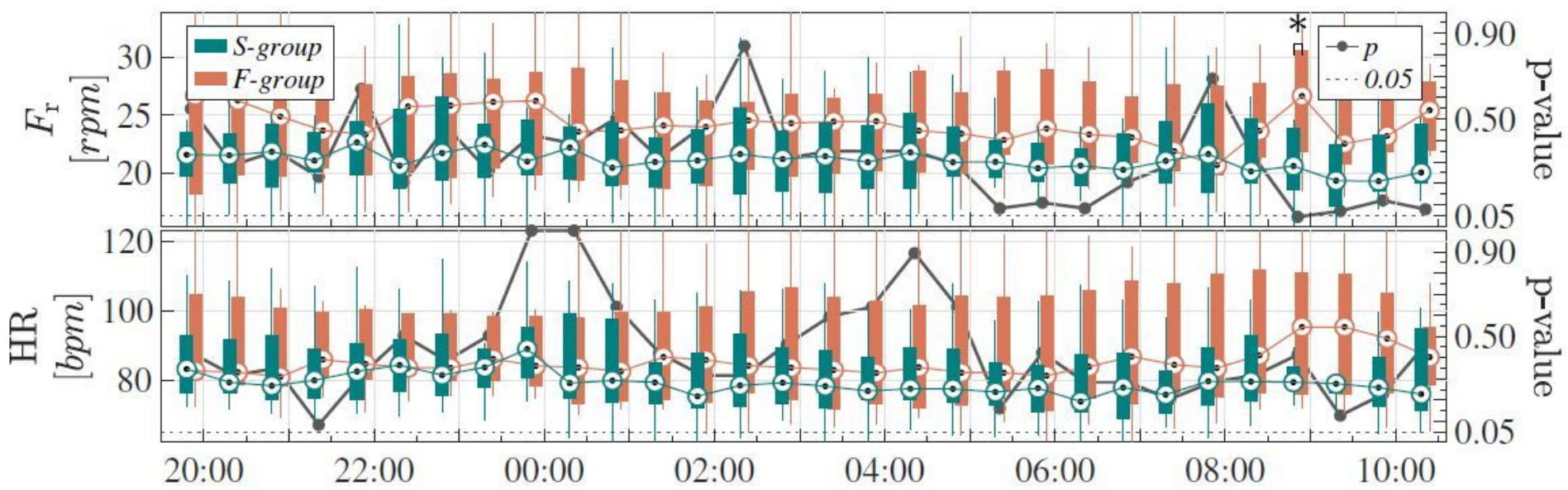

Figure 5 
Evolution of the common clinical indices for weaning readiness before SBT. The mean respiratory frequency, Fr, and mean HR, are represented. Green and Red boxplots represent the patients of the Sgroup and the F-group, respectively. The p-value comparing each half hour is represented in the right axis, from 0 to 1 , and the dotted line represents the $p=0: 05$ threshold. The asterisks indicate statistical significance with $p \leq 0: 05$.
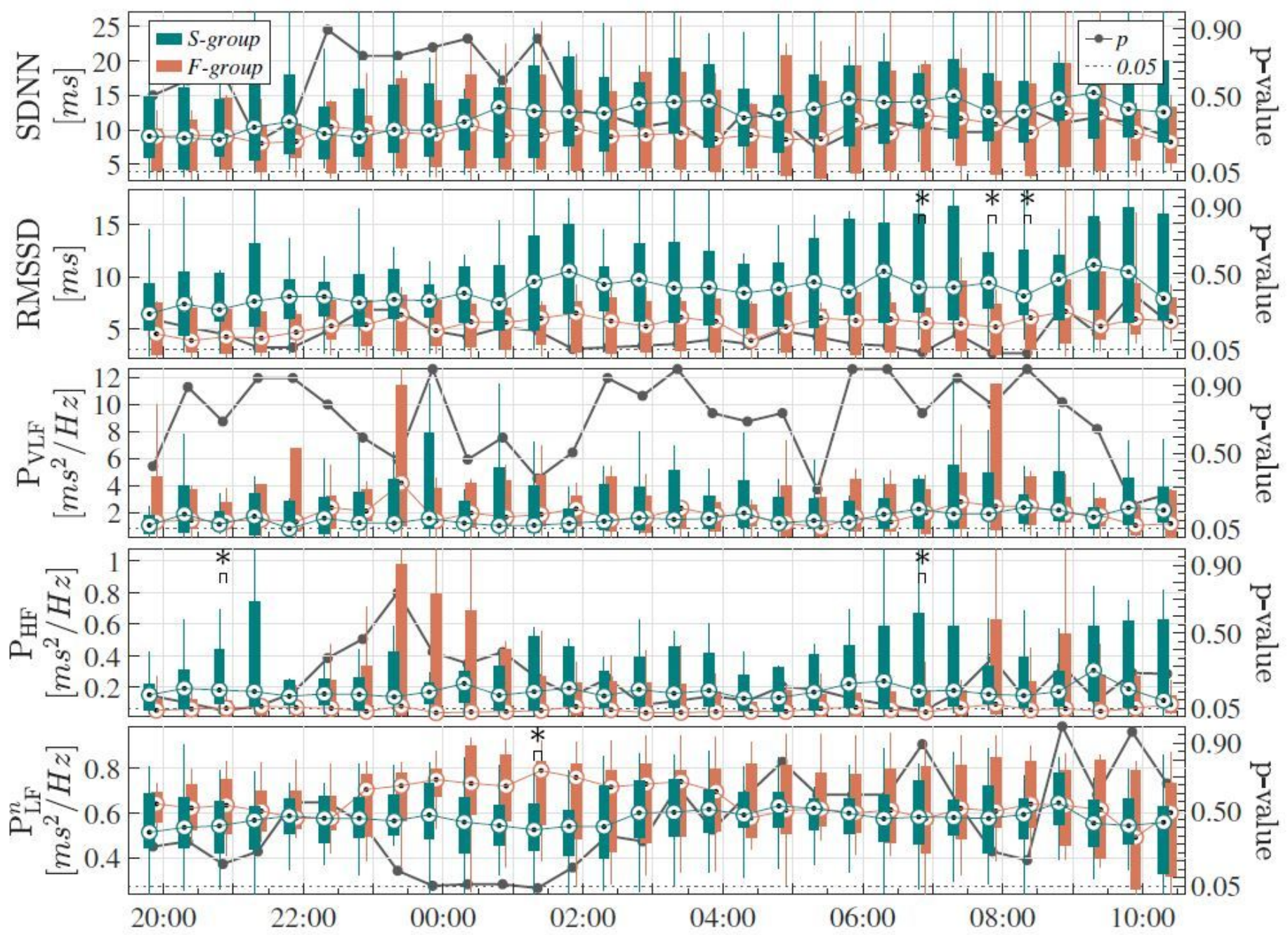

Figure 6

Evolution of the HRV indices before SBT. The temporal parameters SDNN and RMSSD, and the frequency parameters, PVLF, PHF and Pn LF are represented. Green and Red boxplots represent the patients of the S-group and the F-group, respectively. The p-value comparing each half hour is represented in the right axis, from 0 to 1 , and the dotted line represents the $p=0: 05$ threshold. The asterisks indicate statistical significance with $p \leq 0: 05$. 


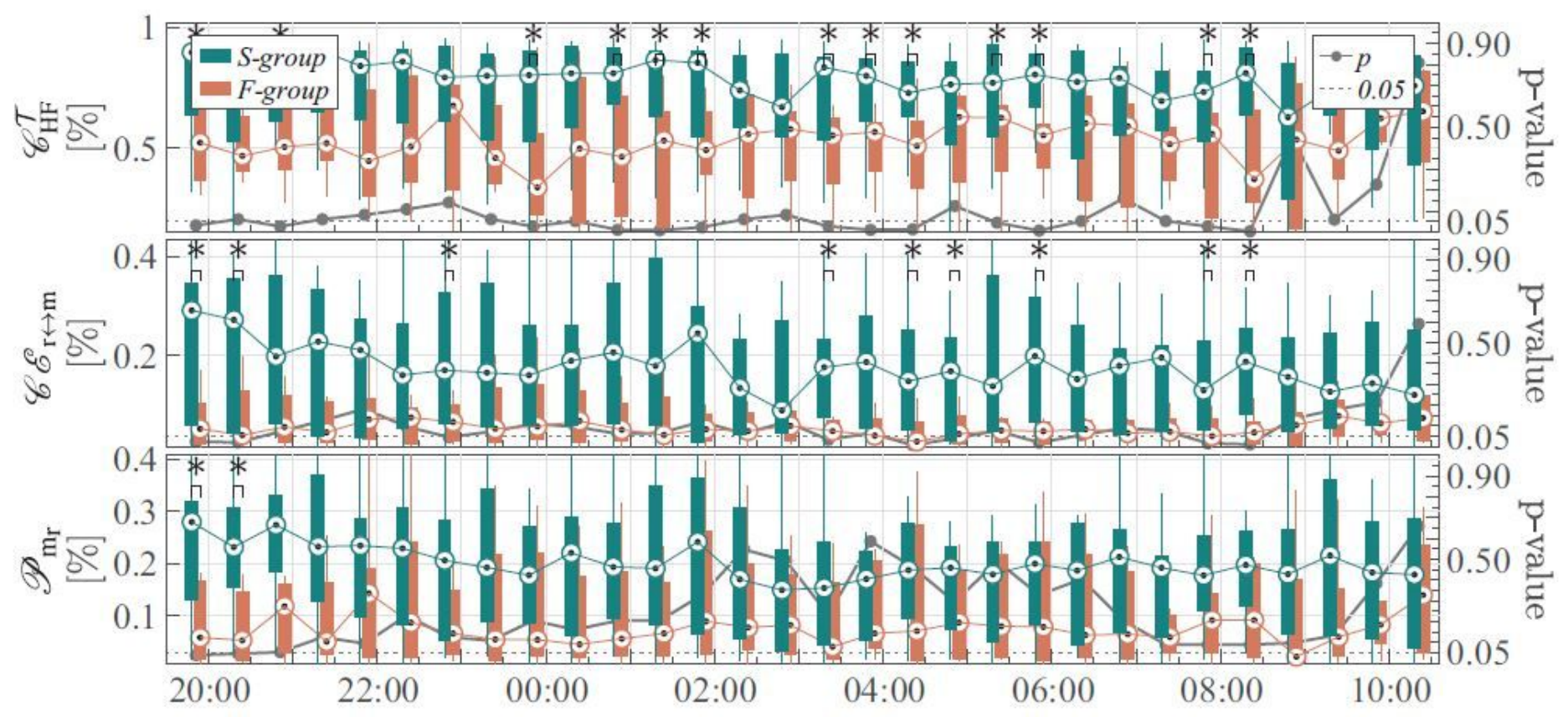

Figure 7

Evolution of the CPC estimators before SBT. The CPC parameters C THF,C E r\m and Pmr, are represented. Green and Red boxplots represent the patients of the S-group and the F-group, respectively. The $p$-value comparing each half hour is represented in the right axis, from 0 to 1 , and the dotted line represents the $p=0: 05$ threshold. The asterisks indicate statistical significance with $p \leq 0: 05$. 